\title{
GLOBAL REGISTRATION OF MULTIPLE POINT CLOUDS USING SEMIDEFINITE PROGRAMMING
}

\author{
KUNAL N. CHAUDHURY, YUEHAW KHOO, AND AMIT SINGER
}

\begin{abstract}
Consider $N$ points in $\mathbb{R}^{d}$ and $M$ local coordinate systems that are related through unknown rigid transforms. For each point we are given (possibly noisy) measurements of its local coordinates in some of the coordinate systems. Alternatively, for each coordinate system, we observe the coordinates of a subset of the points. The problem of estimating the global coordinates of the $N$ points (up to a rigid transform) from such measurements comes up in distributed approaches to molecular conformation and sensor network localization, and also in computer vision and graphics.

The least-squares formulation of this problem, though non-convex, has a well known closed-form solution when $M=2$ (based on the singular value decomposition). However, no closed form solution is known for $M \geq 3$.

In this paper, we demonstrate how the least-squares formulation can be relaxed into a convex program, namely a semidefinite program (SDP). By setting up connections between the uniqueness of this SDP and results from rigidity theory, we prove conditions for exact and stable recovery for the SDP relaxation. In particular, we prove that the SDP relaxation can guarantee recovery under more adversarial conditions compared to earlier proposed spectral relaxations, and derive error bounds for the registration error incurred by the SDP relaxation.

We also present results of numerical experiments on simulated data to confirm the theoretical findings. We empirically demonstrate that (a) unlike the spectral relaxation, the relaxation gap is mostly zero for the semidefinite program (i.e., we are able to solve the original non-convex least-squares problem) up to a certain noise threshold, and (b) the semidefinite program performs significantly better than spectral and manifold-optimization methods, particularly at large noise levels.
\end{abstract}

Keywords: Global registration, rigid transforms, rigidity theory, spectral relaxation, spectral gap, convex relaxation, semidefinite program (SDP), exact recovery, noise stability.

\section{INTRODUCTION}

The problem of point-cloud registration comes up in computer vision and graphics [52, 59, 65], and in distributed approaches to molecular conformation [20, 17] and sensor network localization [16, 9]. The registration problem in question is one of determining the coordinates of a point cloud $P$ from the knowledge of (possibly noisy) coordinates of smaller point cloud subsets (called patches) $P_{1}, \ldots, P_{M}$ that are derived from $P$ through some general transformation. In certain applications [45, 59, 40], one is often interested in finding the optimal transforms (one for each patch) that consistently align $P_{1}, \ldots, P_{M}$. This can be seen as a sub-problem in the determination of the coordinates of $P$ [16, 51].

In this paper, we consider the problem of rigid registration in which the points within a given $P_{i}$ are (ideally) obtained from $P$ through an unknown rigid transform. 
Moreover, we assume that the correspondence between the local patches and the original point cloud is known, that is, we know beforehand as to which points from $P$ are contained in a given $P_{i}$. In fact, one has a control on the correspondence in distributed approaches to molecular conformation [17] and sensor network localization [9, 69, 16]. While this correspondence is not directly available for certain graphics and vision problems, such as multiview registration [49], it is in principle possible to estimate the correspondence by aligning pairs of patches, e.g., using the ICP (Iterative Closest Point) algorithm [6, 51, 36].

1.1. Two-patch Registration. The particular problem of two-patch registration has been well-studied [21, 34, 2]. In the noiseless setting, we are given two point clouds $\left\{x_{1}, \ldots, x_{N}\right\}$ and $\left\{y_{1}, \ldots, y_{N}\right\}$ in $\mathbb{R}^{d}$, where the latter is obtained through some rigid transform of the former. Namely,

$$
y_{k}=O x_{k}+t \quad(k=1, \ldots, N),
$$

where $O$ is some unknown $d \times d$ orthogonal matrix (that satisfies $O^{T} O=I_{d}$ ) and $t \in \mathbb{R}^{d}$ is some unknown translation.

The problem is to infer $O$ and $t$ from the above equations. To uniquely determine $O$ and $t$, one must have at least $N \geq d+1$ non-degenerate point ${ }^{1}$ In this case, $O$ can be determined simply by fixing the first equation in (1) and subtracting (to eliminate $t$ ) any of the remaining $d$ equations from it. Say, we subtract the next $d$ equations:

$$
\left[y_{2}-y_{1} \cdots y_{d+1}-y_{1}\right]=O\left[x_{2}-x_{1} \cdots x_{d+1}-x_{1}\right] .
$$

By the non-degeneracy assumption, the matrix on the right of $O$ is invertible, and this gives us $O$. Plugging $O$ into any of the equations in (1), we get $t$.

In practical settings, (1) would hold only approximately, say, due to noise or model imperfections. A particular approach then would be to determine the optimal $O$ and $t$ by considering the following least-squares program:

$$
\min _{O \in \mathbb{O}(d), t \in \mathbb{R}^{d}} \sum_{k=1}^{N}\left\|y_{k}-O x_{k}-t\right\|_{2}^{2} .
$$

Note that the problem looks difficult a priori since the domain of optimization is $\mathbb{O}(d) \times \mathbb{R}^{d}$, which is non-convex. Remarkably, the global minimizer of this nonconvex problem can be found exactly, and has a simple closed-form expression [19, 39, 32, 21, 34, 2]. More precisely, the optimal $O^{\star}$ is given by $V U^{T}$, where $U \Sigma V^{T}$ is the singular value decomposition (SVD) of

$$
\sum_{k=1}^{N}\left(x_{k}-x_{c}\right)\left(y_{k}-y_{c}\right)^{T},
$$

in which $x_{c}=\left(x_{1}+\cdots+x_{N}\right) / N$ and $y_{c}=\left(y_{1}+\cdots+y_{N}\right) / N$ are the centroids of the respective point clouds. The optimal translation is $t^{\star}=y_{c}-O^{\star} x_{c}$.

The fact that two-patch registration has a closed-form solution is used in the socalled incremental (sequential) approaches for registering multiple patches [6]. The most well-known method is the ICP algorithm [51] (note that ICP uses other heuristics and refinements besides registering corresponding points). Roughly, the idea in sequential registration is to register two overlapping patches at a time,

\footnotetext{
${ }^{1}$ By non-degenerate, we mean that the affine span of the points is $d$ dimensional.
} 
and then integrate the estimated pairwise transforms using some means. The integration can be achieved either locally (on a patch-by-patch basis), or using global cycle-based methods such as synchronization [52, 35, 53, 59, 63]. More recently, it was demonstrated that, by locally registering overlapping patches and then integrating the pairwise transforms using synchronization, one can design efficient and robust methods for distributed sensor network localization [16] and molecular conformation [17]. Note that, while the registration phase is local, the synchronization method integrates the local transforms in a globally consistent manner. This makes it robust to error propagation that often plague local integration methods [35, 63].

1.2. Multi-patch Registration. To describe the multi-patch registration problem, we first introduce some notations. Suppose $x_{1}, x_{2}, \ldots, x_{N}$ are the unknown global coordinates of a point cloud in $\mathbb{R}^{d}$. The point cloud is divided into patches $P_{1}, P_{2}, \ldots, P_{M}$, where each $P_{i}$ is a subset of $\left\{x_{1}, x_{2}, \ldots, x_{N}\right\}$. The patches are in general overlapping, whereby a given point can belong to multiple patches. We represent this membership using an undirected bipartite graph $\Gamma=\left(V_{x} \cup V_{P}, E\right)$. The set of vertices $V_{x}=\left\{x_{1}, \ldots, x_{N}\right\}$ represents the point cloud, while $V_{P}=\left\{P_{1}, \ldots, P_{M}\right\}$ represents the patches. The edge set $E=E(\Gamma)$ connects $V_{x}$ and $V_{P}$, and is given by the requirement that $(k, i) \in E$ if and only if $x_{k} \in P_{i}$. We will henceforth refer to $\Gamma$ as the membership graph.

In this paper, we assume that the local coordinates of a given patch can (ideally) be related to the global coordinates through a single rigid transform, that is, through some rotation, reflection, and translation. More precisely, with every patch $P_{i}$ we associate some (unknown) orthogonal transform $O_{i}$ and translation $t_{i}$. If point $x_{k}$ belongs to patch $P_{i}$, then its representation in $P_{i}$ is given by (cf. (1) and Figure 1)

$$
x_{k, i}=O_{i}^{T}\left(x_{k}-t_{i}\right) \quad(k, i) \in E(\Gamma) .
$$

Alternatively, if we fix a particular patch $P_{i}$, then for every point belonging to that patch,

$$
x_{k}=O_{i} x_{k, i}+t_{i} \quad(k, i) \in E(\Gamma) .
$$

In particular, a given point can belong to multiple patches, and will have a different representation in the coordinate system of each patch.

The premise of this paper is that we are given the membership graph and the local coordinates (referred to as measurements), namely

$$
\Gamma \text { and }\left\{x_{k, i},(k, i) \in E(\Gamma)\right\}
$$

and the goal is to recover the coordinates $x_{1}, \ldots, x_{N}$, and in the process the unknown rigid transforms $\left(O_{1}, t_{1}\right), \ldots,\left(O_{M}, t_{M}\right)$, from (5). Note that the global coordinates are determined up to a global rotation, reflection, and translation. We say that two points clouds (also referred to as configurations) are congruent if one is obtained through a rigid transformation of the other. We will always identify two congruent configurations as being a single configuration.

Under appropriate non-degeneracy assumptions on the measurements, one task would be to specify appropriate conditions on $\Gamma$ under which the global coordinates can be uniquely determined. Intuitively, it is clear that the patches must have enough points in common for the registration problem to have an unique solution. 


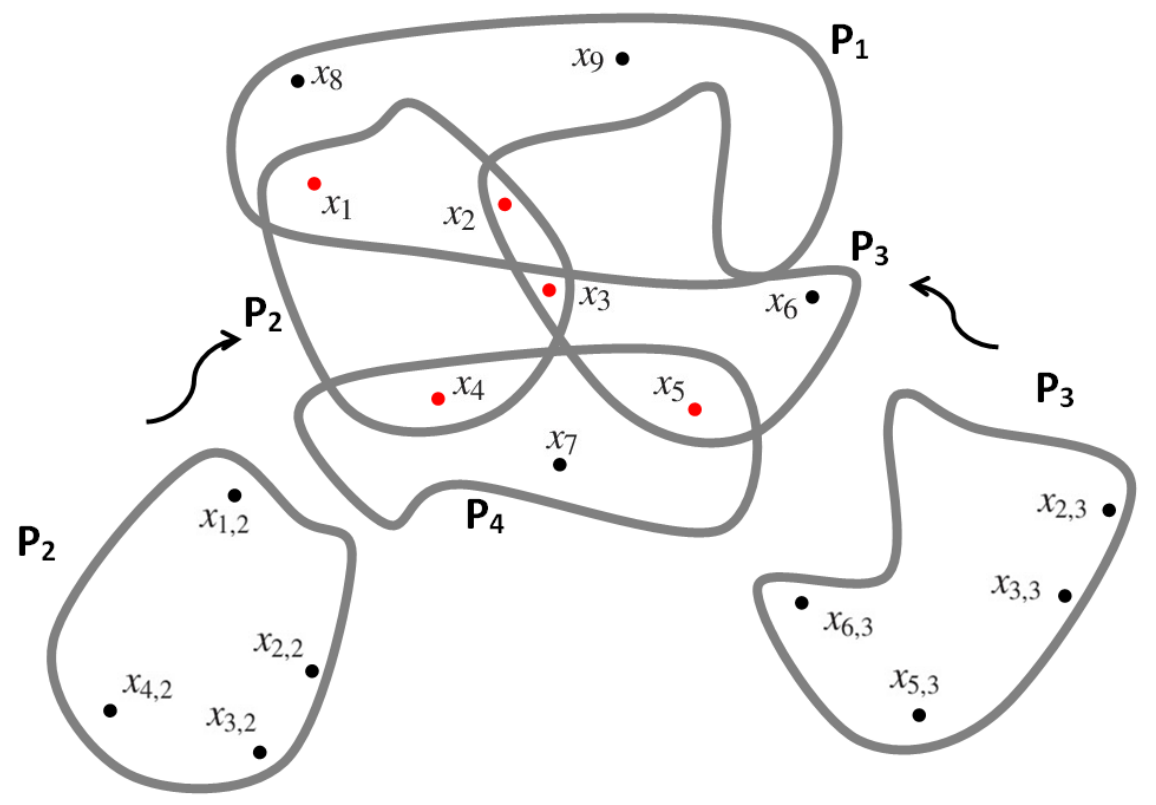

FIGURE 1 . The problem of registering 3 patches on $\mathbb{R}^{2}$, where one is required to find the global coordinates of the points from the corresponding local patch coordinates. The local coordinates of the points in patches $P_{2}$ and $P_{3}$ are shown (see (5) for the notation of local coordinates). It is the common points belonging to two or more patches (marked in red) that contribute to the registration. Note that sequential or pairwise registration would fail in this case. This is because no pair of patches can be registered as they have less than 3 points in common (at least 3 points are required to fix rotations, reflections, and translations in $\mathbb{R}^{2}$ ). The SDP-based algorithm proposed in this paper does a global registration, and is able to recover the exact global coordinates for this example.

For example, it is clear that the global coordinates cannot be uniquely recovered if $\Gamma$ is disconnected.

In practical applications, we are confronted with noisy settings where (4) holds only approximately. In such cases, we would like to determine the global coordinates and the rigid transforms such that the discrepancy in (4) is minimal. In particular, we consider the following quadratic loss:

$$
\phi=\sum_{(k, i) \in E(\Gamma)}\left\|x_{k}-O_{i} x_{k, i}-t_{i}\right\|^{2}
$$


where $\|\cdot\|$ is the Euclidean norm on $\mathbb{R}^{d}$. The optimization problem is to minimize $\phi$ with respect to the following variables:

$$
x_{1}, x_{2}, \ldots, x_{N} \in \mathbb{R}^{d}, \quad O_{1}, \ldots, O_{M} \in \mathbb{O}(d), \quad t_{1}, \ldots, t_{M} \in \mathbb{R}^{d} .
$$

The input to the problem are the measurements in (5). Note that our ultimate goal is to determine $x_{1}, x_{2}, \ldots, x_{N}$; the rigid transforms can be seen as latent variables.

The problem of multipatch registration is intrinsically non-convex since one is required to optimize over the non-convex domain of orthogonal transforms. Different ideas from the optimization literature have been deployed to attack this problem, including Lagrangian optimization and projection methods. In the Lagrangian setup, the orthogonality constraints are incorporated into the objective; in the projection method, the constraints are forced after every step of the optimization [49]. Following the observation that the registration problem can be viewed as an optimization on the Grassmanian and Stiefel manifolds, researchers have proposed algorithms using ideas from the theory and practice of manifold optimization [40]. A detailed review of these methods is beyond the scope of this paper, and instead we refer the interested reader to these excellent reviews [18, 1]. Manifold-based methods are, however, local in nature, and are not guaranteed to find the global minimizer. Moreover, it is rather difficult to certify the noise stability of such methods.

1.3. Contributions. The main contributions of the paper can be organized into the following categories.

(1) Algorithm: We demonstrate how the translations can be factored out of (6), whereby the least-squares problem can be reduced to the following optimization:

$$
\max _{O_{1}, \ldots, O_{M}} \sum_{i, j=1}^{M} \operatorname{Tr}\left(O_{i} C_{i j} O_{j}^{T}\right) \quad \text { subject to } \quad O_{1}, \ldots, O_{M} \in \mathbb{O}(d),
$$

where $C_{i j} \in \mathbb{R}^{d \times d}(1 \leq i, j \leq M)$ are the $(i, j)$-th sub-blocks of some positive semidefinite block matrix $C$ of size $M d \times M d$. Given the solution of (7), the desired global coordinates can simply be obtained by solving a linear system. It is virtually impossible to find the global optimum of (7) for large-scale problems $(M \gg 1)$, since this involves the optimization of a quadratic cost on a huge non-convex parameter space. In fact, the simplest case $d=1$ with $C$ as the Laplacian matrix corresponds to the MAX-CUT problem, which is known to be NP-hard. The main observation of this paper is that (7) can instead be relaxed into a convex program, namely a semidefinite program, whose global optimum can be approximated to any finite precision in polynomial time using standard off-the-shelf solvers. This yields a tractable method for global registration described in Algorithm 2 The corresponding algorithm derived from the spectral relaxation of (7) that was already considered in [40] is described in Algorithm 1 for reference.

(2) Exact Recovery: We present conditions on the coefficient matrix $C$ in (7) for exact recovery using Algorithm 2 In particular, we show that the exact 
recovery questions about Algorithm 2 can be mapped into rigidity theoretic questions that have already been investigated earlier ${ }^{2}$ in [68, 25]. The contribution of this section is the connection made between the $C$ matrix in (7) and various notions of rigidity considered in these papers. We also present an efficient randomized rank test for $C$ than can be used to certify exact recovery (motivated by the work in [31, 26, 54]).

(3) Stability Analysis: We study the stability of Algorithms 1 and 2 for the noise model in which the patch coordinates are perturbed using noise of bounded size (note that the stability of the spectral relaxation was not investigated in [40]). Our main result here is Theorem 13 which states that, if $C$ satisfies a particular rank condition, then the registration error for Algorithm 2 is within a constant factor of the noise level. To the best of our knowledge, there is no existing algorithm for multipatch registration that comes with a similar stability guarantee.

(4) Empirical Results: We present numerical results on simulated data to numerically verify the exact recovery and noise stability properties of Algorithms 1 and 2 Our main empirical findings are the following:

(1) The semidefinite relaxation performs significantly better than spectral and manifold-based optimization (say, with the spectral solution as initialization) in terms of the reconstruction quality (cf. first plot in Figure 7).

(2) Up to a certain noise level, we are actually able to solve the original non-convex problem using the semidefinite relaxation (cf. second plot in Figure 7).

1.4. Broader Context and Related Work. The objective (6) is a straightforward extension of the objective for two-patches [19, 21, 34, 2]. In fact, this objective was earlier considered by Zhang et al. for distributed sensor localization [69]. The present work is also closely tied to the work of Cucuringu et al. on distributed localization [16, 17], where a similar objective is implicitly optimized. The common theme in these works is that some form of optimization is used to globally register the patches, once their local coordinates have been determined by some means. There is, however, some fundamental differences between the various algorithms used to actually perform the optimization. Zhang et al. [69] use alternating leastsquares to iteratively optimize over the global coordinates and the transforms, which to the best of our knowledge has no convergence guarantee. On the other hand, Cucuringu et al. [16, 17] first optimize over the orthogonal transforms (using synchronization [53]), and then solve for the translations (in effect, the global coordinates) using least-squares fitting. In this work, we combine these different ideas into a single framework. While our objective is similar to the one used in [69], we jointly optimize the rigid transforms and positions. In particular, the algorithms considered in Section 2 avoid the convergence issues associated with alternating least-squares in [69], and are able to register patch systems that cannot be registered using the approach in [16, 17].

\footnotetext{
${ }^{2}$ The authors thank the anonymous referees for pointing this out.
} 
Another closely related work is the paper by Krishnan et al. on global registration [40], where the optimal transforms (rotations to be specific) are computed by extending the objective in (2) to the multipatch case. The subsequent mathematical formulation has strong resemblance with our formulation, and, in fact, leads to a subproblem similar to (7). Krishnan et al. [40] propose the use of manifold optimization to solve (7), where the manifold is the product manifold of rotations. However, as mentioned earlier, manifold methods generally do not offer guarantees on convergence (to the global minimum) and stability. Moreover, the manifold in (7) is not connected. Therefore, any local method will fail to attain the global optimum of (7) if the initial guess is on the wrong component of the manifold.

It is exactly at this point that we depart from [40], namely, we propose to relax (7) into a tractable semidefinite program (SDP). This was motivated by a long line of work on the use of SDP relaxations for non-convex (particularly NP-hard) problems. See, for example, [43, 23, 66, 47, 12, 41], and these reviews [60, 48, 70]. Note that for $d=1,(7)$ is a quadratic Boolean optimization, similar to the MAX-CUT problem. An SDP-based algorithm with randomized rounding for solving MAX-CUT was proposed in the seminal work of Goemans and Williamson [23]. The semidefinite relaxation that we consider in Section 2 is motivated by this work. In connection with the present work, we note that provably stable SDP algorithms have been considered for low rank matrix completion [12], phase retrieval [13, 62], and graph localization [37].

We note that a special case of the registration problem considered here is the socalled generalized Procrustes problem [28]. Within the point-patch framework just introduced, the goal in Procrustes analysis is to find $O_{1}, \ldots, O_{M} \in \mathbb{O}(d)$ that minimize

$$
\sum_{k=1}^{N} \sum_{i, j=1}^{M}\left\|O_{i} x_{k, i}-O_{j} x_{k, j}\right\|^{2} .
$$

In other words, the goal is to achieve the best possible alignment of the $M$ patches through orthogonal transforms. This can be seen as an instance of the global registration problem without the translations $\left(t_{1}=\cdots=t_{M}=0\right)$, and in which $\Gamma$ is complete. It is not difficult to see that (8) can be reduced to (7). On the other hand, using the analysis in Section 2 , it can be shown that (6) is equivalent to $(8)$ in this case. While the Procrustes problem is known to be NP-hard, several polynomialtime approximations with guarantees have been proposed. In particular, SDP relaxations of (8) have been considered in [47, 55, 46], and more recently in [3]. We use the relaxation of (7) considered in [3] for reasons to be made precise in Section 2 .

1.5. Notations. We use upper case letters such as $O$ to denote matrices, and lower case letters such as $t$ for vectors. We use $I_{d}$ to denote the identity matrix of size $d \times d$. We denote the diagonal matrix of size $n \times n$ with diagonal elements $c_{1}, \ldots, c_{n}$ as $\operatorname{diag}\left(c_{1}, \ldots, c_{n}\right)$. We will frequently use block matrices built from smaller matrices, typically of size $d \times d$, where $d$ is the dimension of the ambient space. For some block matrix $A$, we will use $A_{i j}$ to denote its $(i, j)$-th block, and $A(p, q)$ to denote its $(p, q)$-th entry. In particular, if each block has size $d \times d$, then

$$
A_{i j}(p, q)=A((i-1) d+p,(j-1) d+q) \quad(1 \leq p, q \leq d) .
$$


We use $A \succeq 0$ to mean that $A$ is positive semidefinite, that is, $u^{T} A u \geq 0$ for all $u$. We use $\mathbb{O}(d)$ to denote the group of orthogonal transforms (matrices) acting on $\mathbb{R}^{d}$, and $\mathbb{O}(d)^{M}$ to denote the $M$-fold product of $\mathbb{O}(d)$ with itself. We will also conveniently identify the matrix $\left[O_{1} \cdots O_{M}\right]$ with an element of $\mathbb{O}(d)^{M}$ where each $O_{i} \in \mathbb{O}(d)$. We use $\|x\|$ to denote the Euclidean norm of $x \in \mathbb{R}^{n}$ ( $n$ will usually be clear from the context, and will be pointed out if this is not so). We denote the trace of a square matrix $A$ by $\operatorname{Tr}(A)$. The Frobenius and spectral norms are defined as

$$
\|A\|_{F}=\operatorname{Tr}\left(A^{T} A\right)^{1 / 2} \text { and }\|A\|_{\mathrm{sp}}=\max _{\|x\| \leq 1}\|A x\| .
$$

The Kronecker product between matrices $A$ and $B$ is denoted by $A \otimes B$ [24]. The all-ones vector is denoted by $e$ (the dimension will be obvious from the context), and $e_{i}^{N}$ denotes the all-zero vector of length $N$ with 1 at the $i$-th position.

1.6. Organization. In the next section, we present the semidefinite relaxation of the least-squares registration problem described in the introduction. For reference, we also present the closely related spectral relaxation that was already considered in [40, 68, 25]. Exact recovery questions are addressed in section 3, followed by a randomized test in section 4 Stability analysis for the spectral and semidefinite relaxations are presented in section 5 Numerical simulations can be found in section 6 and a discussion of certain open questions in section 7

\section{Spectral AND Semidefinite Relaxations}

The minimization of (6) involves unconstrained variables (global coordinates and patch translations) and constrained variables (the orthogonal transformations). We first solve for the unconstrained variables in terms of the unknown orthogonal transformations, representing the former as linear combinations of the latter. This reduces (6) to a quadratic optimization problem over the orthogonal transforms of the form (7).

In particular, we combine the global coordinates and the translations into a single matrix:

$$
Z=\left[x_{1} \cdots x_{N} t_{1} \cdots t_{M}\right] \in \mathbb{R}^{d \times(N+M)} .
$$

Similarly, we combine the orthogonal transforms into a single matrix,

$$
O=\left[O_{1} \cdots O_{M}\right] \in \mathbb{R}^{d \times M d} .
$$

Recall that we will conveniently identify $O$ with an element of $\mathbb{O}(d)^{M}$.

To express (6) in terms of $Z$ and $O$, we write $x_{k}-t_{i}=Z e_{k i}$, where

$$
e_{k i}=e_{k}^{N+M}-e_{N+i}^{N+M} \text {. }
$$

Similarly, we write $O_{i}=O\left(e_{i}^{M} \otimes I_{d}\right)$. This gives us

$$
\phi(Z, O)=\sum_{(k, i) \in E(\Gamma)}\left\|Z e_{k i}-O\left(e_{i}^{M} \otimes I_{d}\right) x_{k, i}\right\|^{2} .
$$

Using $\|x\|^{2}=\operatorname{Tr}\left(x x^{T}\right)$, and properties of the trace, we obtain

$$
\phi(Z, O)=\operatorname{Tr}\left(\left[\begin{array}{ll}
Z & O
\end{array}\right]\left[\begin{array}{cc}
L & -B^{T} \\
-B & D
\end{array}\right]\left[\begin{array}{l}
Z^{T} \\
O^{T}
\end{array}\right]\right),
$$


where

$$
\begin{aligned}
L & =\sum_{(k, i) \in E} e_{k i} e_{k i}^{T}, \quad B=\sum_{(k, i) \in E}\left(e_{i}^{M} \otimes I_{d}\right) x_{k, i} e_{k i}^{T}, \text { and } \\
D & =\sum_{(k, i) \in E}\left(e_{i}^{M} \otimes I_{d}\right) x_{k, i} x_{k, i}{ }^{T}\left(e_{i}^{M} \otimes I_{d}\right)^{T} .
\end{aligned}
$$

The matrix $L$ is the combinatorial graph Laplacian of $\Gamma$ [14], and is of size $(N+$ $M) \times(N+M)$. The matrix $B$ is of size $M d \times(N+M)$, and the size of the block diagonal matrix $D$ is $M d \times M d$.

The optimization program now reads

$$
\min _{Z, O} \phi(Z, O) \quad \text { subject to } Z \in \mathbb{R}^{d \times(N+M)}, O \in \mathbb{O}(d)^{M} .
$$

The fact that $\mathbb{O}(d)^{M}$ is non-convex makes (P) non-convex. In the next few subsections, we will show how this non-convex program can be approximated by tractable spectral and convex programs.

2.1. Optimization over translations. Note that we can write $(\mathrm{P})$ as

$$
\min _{O \in \mathbb{O}(d)^{M}}\left[\min _{Z \in \mathbb{R}^{d \times(N+M)}} \phi(Z, O)\right] .
$$

That is, we first minimize over the free variable $Z$ for some fixed $O \in \mathbb{O}(d)^{M}$, and then we minimize with respect to $O$.

Fix some arbitrary $O \in \mathbb{O}(d)^{M}$, and set $\psi(Z)=\phi(Z, O)$. It is clear from (11) that $\psi(Z)$ is quadratic in $Z$. In particular, the stationary points $Z^{\star}=Z^{\star}(O)$ of $\psi(Z)$ satisfy

$$
\nabla \psi\left(Z^{\star}\right)=0 \quad \Rightarrow \quad Z^{\star} L=O B .
$$

The Hessian of $\psi(Z)$ equals $2 L$, and it is clear from (12) that $L \succeq 0$. Therefore, $Z^{\star}$ is a minimizer of $\psi(Z)$.

If $\Gamma$ is connected, then $e$ is the only vector in the null space of $L[14]$. Let $L^{\dagger}$ be the Moore-Penrose pseudo-inverse of $L$, which is again positive semidefinite. It can be verified that

$$
L L^{\dagger}=L^{\dagger} L=I_{N+M}-(N+M)^{-1} e e^{T} .
$$

If we right multiply (13) by $L^{\dagger}$, we get

$$
Z^{\star}=O B L^{\dagger}+t e^{T}
$$

where $t \in \mathbb{R}^{d}$ is some global translation. Conversely, if we right multiply (15) by $L$ and use the facts that $e^{T} L=0$ and $B e=0$, we get (13). Thus, every solution of (13) is of the form (15).

Substituting (15) into (11), we get

$$
\psi\left(Z^{\star}\right)=\phi\left(Z^{\star}, O\right)=\operatorname{Tr}\left(C O^{T} O\right)=\sum_{i, j=1}^{M} \operatorname{Tr}\left(O_{i} C_{i j} O_{j}^{T}\right),
$$

where

$$
C=\left[\begin{array}{ll}
B L^{\dagger} & I_{M d}
\end{array}\right]\left[\begin{array}{cc}
L & -B^{T} \\
-B & D
\end{array}\right]\left[\begin{array}{c}
L^{\dagger} B^{T} \\
I_{M d}
\end{array}\right]=D-B L^{\dagger} B^{T}
$$


Note that (16) has the global translation $t$ taken out. This is not a surprise since $\phi$ is invariant to global translations. Moreover, note that we have not forced the orthogonal constraints on $O$ as yet. Since $\phi(Z, O) \geq 0$ for any $Z$ and $O$, it necessarily follows from (16) that $C \succeq 0$. We will see in the sequel how the spectrum of $C$ dictates the performance of the convex relaxation of (16).

In analogy with the notion of stress in rigidity theory [26], we can consider [6] as a sum of the "stress" between pairs of patches when we try to register them using rigid transforms. In particular, the $(i, j)$-th term in (16) can be regarded as the stress between the (centered) $i$-th and $j$-th patches generated by the orthogonal transforms. Keeping this analogy in mind, we will henceforth refer to $C$ as the patch-stress matrix.

2.2. Optimization over orthogonal transforms. The goal now is to optimize (16) with respect to the orthogonal transforms, that is, we have reduced $(\mathrm{P})$ to the following problem:

$\left(\mathrm{P}_{0}\right) \quad \min _{O \in \mathbb{R}^{d \times M d}} \operatorname{Tr}\left(C O^{T} O\right) \quad$ subject to $\quad\left(O^{T} O\right)_{i i}=I_{d}(1 \leq i \leq M)$.

This is a non-convex problem since $O$ lives on a non-convex (disconnected) manifold [1]. We will generally refer to any method which uses manifold optimization to solve $\left(\mathrm{P}_{0}\right)$ and then computes the coordinates using (15) as "Global Registration over Euclidean Transforms using Manifold Optimization" (GRET-MANOPT).

2.3. Spectral relaxation and rounding. Following the quadratic nature of the objective in $\left(\mathrm{P}_{0}\right)$, it is possible to relax it into a spectral problem. More precisely, consider the domain

$$
\mathcal{S}=\left\{O \in \mathbb{R}^{d \times M d}: \text { rows of } O \text { are orthogonal and each row has norm } \sqrt{M}\right\} .
$$

That is, we do not require the $d \times d$ blocks in $O \in \mathcal{S}$ to be orthogonal. Instead, we only require the rows of $O$ to form an orthogonal system, and each row to have the same norm. It is clear that $\mathcal{S}$ is a larger domain than that determined by the constraints in $\left(\mathrm{P}_{0}\right)$. In particular, we consider the following relaxation of $\left(\mathrm{P}_{0}\right)$ :

$$
\left(\mathrm{P}_{1}\right) \quad \min _{O \in \mathcal{S}} \operatorname{Tr}\left(C O^{T} O\right) \text {. }
$$

This is precisely a spectral problem in that the global minimizers are determined from the spectral decomposition of $C$. More precisely, let $\mu_{1} \leq \ldots \leq \mu_{M d}$ be eigenvalues of $C$, and let $r_{1}, \ldots, r_{M d}$ be the corresponding eigenvectors. Define

$$
W^{\star} \stackrel{\text { def }}{=} \sqrt{M}\left[r_{1} \cdots r_{d}\right]^{T} \in \mathbb{R}^{d \times M d} .
$$

Then

$$
\operatorname{Tr}\left(C W^{\star T} W^{\star}\right)=\min _{O \in \mathcal{S}} \operatorname{Tr}\left(C O^{T} O\right)=M\left(\mu_{1}+\cdots+\mu_{d}\right) .
$$

Due to the relaxation, the blocks of $W^{\star}$ are not guaranteed to be in $\mathbb{O}(d)$. We round each $d \times d$ block of $W^{\star}$ to its "closest" orthogonal matrix. More precisely, let $W^{\star}=\left[W_{1}^{\star} \cdots W_{M}^{\star}\right]$. For every $1 \leq i \leq M$, we find $O_{i}^{\star} \in \mathbb{O}(d)$ such that

$$
\left\|O_{i}^{\star}-W_{i}^{\star}\right\|_{F}=\min _{O \in \mathbb{O}(d)}\left\|O-W_{i}^{\star}\right\|_{F} .
$$


As noted earlier, this has a closed-form solution, namely $O_{i}^{\star}=U V^{T}$, where $U \Sigma V^{T}$ is the SVD of $W_{i}^{\star}$. We now put the rounded blocks back into place and define

$$
O^{\star} \stackrel{\text { def }}{=}\left[O_{1}^{\star} \ldots O_{M}^{\star}\right] \in \mathbb{O}(d)^{M} .
$$

In the final step, following (15), we define

$$
Z^{\star} \stackrel{\text { def }}{=} O^{\star} B L^{\dagger} \in \mathbb{R}^{d \times(N+M)} .
$$

The first $N$ columns of $Z^{\star}$ are taken to be the reconstructed global coordinates.

We will refer to this spectral method as the "Global Registration over Euclidean Transforms using Spectral Relaxation" (GRET-SPEC). The main steps of GRET-SPEC are summarized in Algorithm 1. We note that a similar spectral algorithm was proposed for angular synchronization by Bandeira et al. [4], and by Krishnan et al. [40] for initializing the manifold optimization.

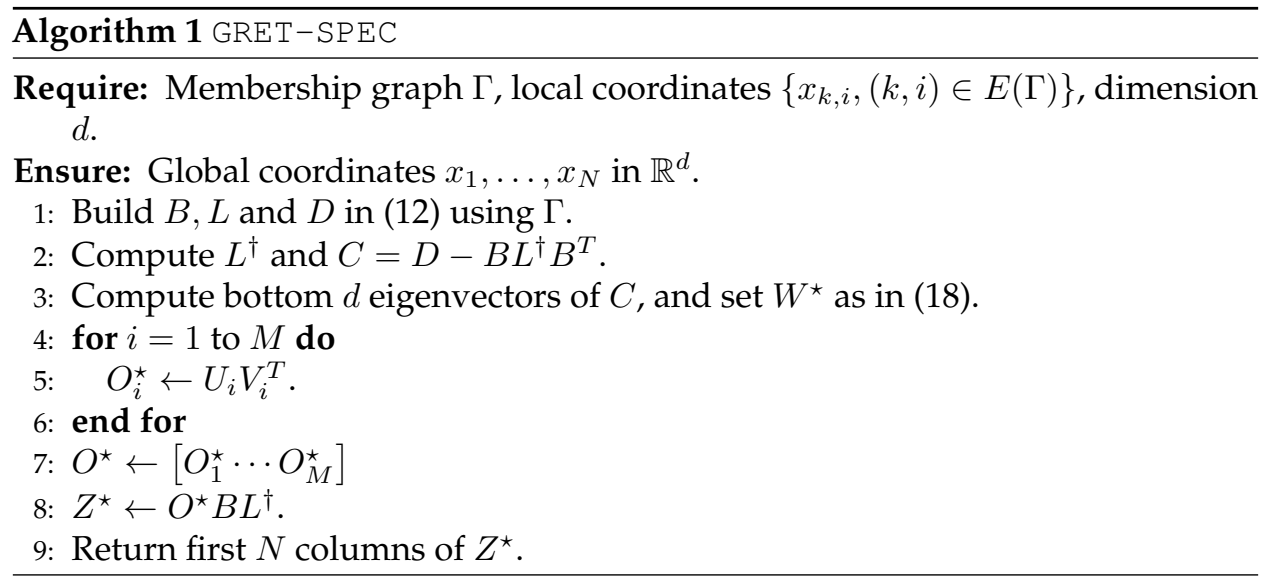

The question at this point is how are the quantities $O^{\star}$ and $Z^{\star}$ obtained from GRET-SPEC related to the original problem $(\mathrm{P})$ ? Since $\left(\mathrm{P}_{1}\right)$ is obtained by relaxing the block-orthogonality constraint in $\left(\mathrm{P}_{0}\right)$, it is clear that if the blocks of $W^{\star}$ are orthogonal, then $O^{\star}$ and $Z^{\star}$ are solutions of $(\mathrm{P})$, that is,

$$
\phi\left(Z^{\star}, O^{\star}\right) \leq \phi(Z, O) \quad \text { for all } Z \in \mathbb{R}^{d \times(N+M)}, O \in \mathbb{O}(d)^{M} .
$$

We have actually found the global minimizer of the original non-convex problem $(\mathrm{P})$ in this case.

Observation 1 (Tight relaxation using GRET-SPEC). If the $d \times d$ blocks of the solution of $\left(\mathrm{P}_{1}\right)$ are orthogonal, then the coordinates and transforms computed by GRET-SPEC are the global minimizers of $(\mathrm{P})$.

If some the blocks are not orthogonal, the rounded quantities $O^{\star}$ and $Z^{\star}$ are only an approximation of the solution of $(\mathrm{P})$.

2.4. Semidefinite relaxation and rounding. We now explain how we can obtain a tighter relaxation of $\left(\mathrm{P}_{0}\right)$ using a semidefinite program, for which the global minimizer can be computed efficiently. Our semidefinite program was motivated by the line of works on the semidefinite relaxation of non-convex problems [43, 23. 60, 12]. 
Consider the domain

$$
\mathcal{C}=\left\{O \in \mathbb{R}^{M d \times M d}:\left(O^{T} O\right)_{11}=\cdots=\left(O^{T} O\right)_{M M}=I_{d}\right\} .
$$

That is, while we require the columns of each $M d \times d$ block of $O \in \mathcal{C}$ to be orthogonal, we do not force the non-convex $\operatorname{rank}$ constraint $\operatorname{rank}(O)=d$. This gives us the following relaxation

$$
\min _{O \in \mathcal{C}} \operatorname{Tr}\left(C O^{T} O\right) .
$$

Introducing the variable $G=O^{T} O, 22$ is equivalent to

$\left(\mathrm{P}_{2}\right) \quad \min _{G \in \mathbb{R}^{M d \times M d}} \operatorname{Tr}(C G) \quad$ subject to $G \succeq 0, G_{i i}=I_{d}(1 \leq i \leq M)$.

This is a standard semidefinite program [60] which can be solved using software packages such as SDPT3 [58] and CVX [29]. We provide details about SDP solvers and their computational complexity later in Section 2.5

Let us denote the solution of $\left(\mathrm{P}_{2}\right)$ by $G^{\star}$, that is,

$$
\operatorname{Tr}\left(C G^{\star}\right)=\min _{G \in \mathbb{R}^{M d \times M d}}\left\{\operatorname{Tr}(C G): G \succeq 0, G_{11}=\cdots=G_{M M}=I_{d}\right\} .
$$

By the linear constraints in $\left(\mathrm{P}_{2}\right)$, it follows that $\operatorname{rank}\left(G^{\star}\right) \geq d$. If $\operatorname{rank}\left(G^{\star}\right)>d$, we need to round (approximate) it by a rank- $d$ matrix. That is, we need to project it onto the domain of $\left(\mathrm{P}_{0}\right)$. One possibility would be to use random rounding that come with approximation guarantees; for example, see [23, 3]. In this work, we use deterministic rounding, namely the eigenvector rounding which retains the top $d$ eigenvalues and discards the remaining. In particular, let $\lambda_{1} \geq \lambda_{2} \geq \cdots \geq \lambda_{M d}$ be the eigenvalues of $G^{\star}$, and $q_{1}, \ldots, q_{M d}$ be the corresponding eigenvectors. Let

$$
W^{\star} \stackrel{\text { def }}{=}\left[\sqrt{\lambda_{1}} q_{1} \cdots \sqrt{\lambda_{d}} q_{d}\right]^{T} \in \mathbb{R}^{d \times M d} .
$$

We now proceed as in the GRET-SPEC, namely, we define $O^{\star}$ and $Z^{\star}$ from $W^{\star}$ as in 20) and (21). We refer to the complete algorithm as "Global Registration over Euclidean Transforms using SDP" (GRET-SDP). The main steps of GRET-SDP are summarized in Algorithm 2

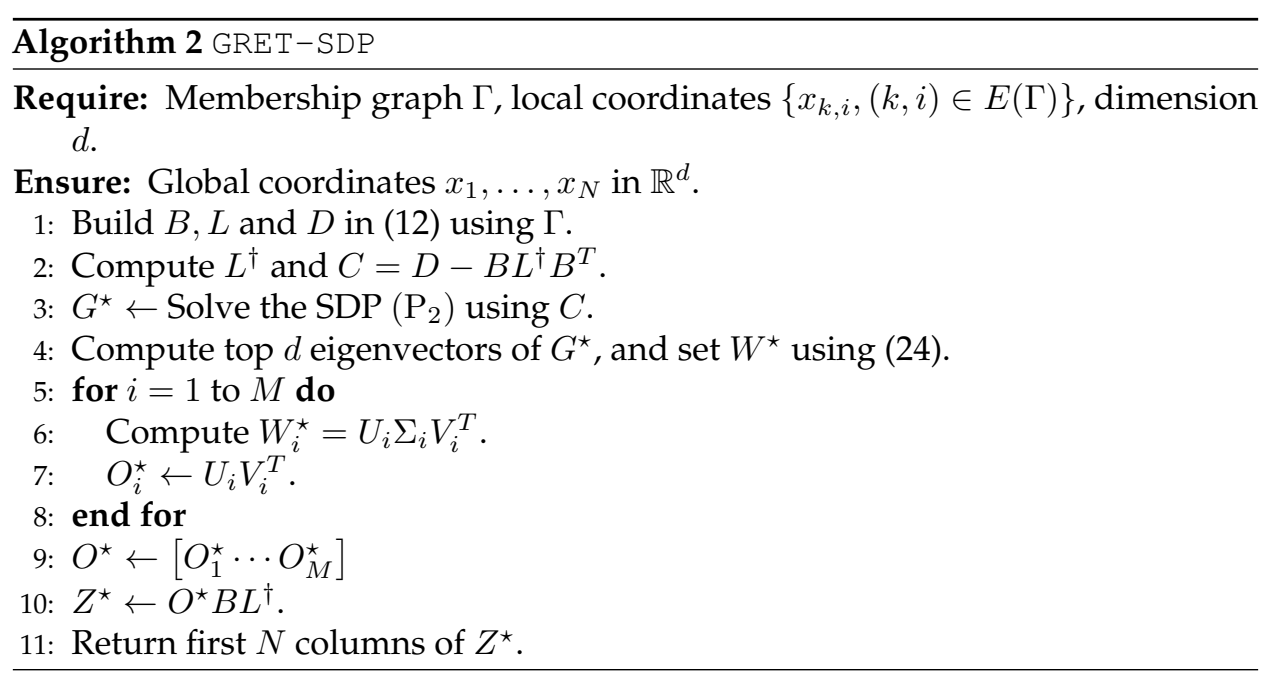

Similar to Observation 1 , we note the following for GRET-SDP. 
Observation 2 (Tight relaxation using GRET-SDP). If the rank of the solution of $\left(\mathrm{P}_{2}\right)$ is exactly $d$, then the coordinates and transforms computed by GRET-SDP are the global minimizers of $(\mathrm{P})$.

If $\operatorname{rank}\left(G^{\star}\right)>d$, the output of GRET-SDP can only be considered as an approximation of the solution of $(\mathrm{P})$. The quality of the approximation for $\left(\mathrm{P}_{2}\right)$ can be quantified using, for example, the randomized rounding in [3]. More precisely, note that since $D$ is block-diagonal, (22) is equivalent (up to a constant term) to

$$
\max _{O \in \mathcal{C}} \operatorname{Tr}\left(Q O^{T} O\right)
$$

where $Q=B L^{\dagger} B^{T} \succeq 0$. Bandeira et al. [3] show that the orthogonal transforms (which we continue to denote by $O^{\star}$ ) obtained by a certain random rounding of $G^{\star}$ satisfy

$$
\mathbb{E}\left[\operatorname{Tr}\left(Q O^{\star T} O^{\star}\right)\right] \geq \alpha_{d}^{2} \cdot \mathrm{OPT},
$$

where OPT is the optimum of the unrelaxed problem (7) with $Q=B L^{\dagger} B^{T}$, and $\alpha_{d}$ is the expected average of the singular values of a $d \times d$ random matrix with entries iid $\mathcal{N}(0,1 / d)$. It was conjectured in [3] that $\alpha_{d}$ is monotonically increasing, and the boundary values were computed to be $\alpha_{1}=\sqrt{2 / \pi}$ ( $\alpha_{1}$ was also reported here [48]) and $\alpha_{\infty}=8 / 3 \pi$. We refer the reader to [3] for further details on the rounding procedure, and its relation to previous work in terms of the approximation ratio. Empirical results, however, suggest that the difference between deterministic and randomized rounding is small as far as the final reconstruction is concerned. We will therefore simply use the deterministic rounding.

2.5. Computational complexity. The main computations in GRET-SPEC are the Laplacian inversion, the eigenvector computation, and the orthogonal rounding. The cost of inverting $L$ when $\Gamma$ is dense is $O\left((N+M)^{3}\right)$. However, for most practical applications, we expect $\Gamma$ to be sparse since every point would typically be contained in a small number of patches. In this case, it is known that the linear system $L x=b$ can be solved in time almost linear in the number of edges in $\Gamma$ [57, 61]. Applied to (14), this means that we can compute $L^{\dagger}$ in $O(|E(\Gamma)|)$ time (up to logarithmic factors). Note that, even if $L$ is dense, it is still possible to speed up the inversion (say, compared to a direct Gaussian elimination) using the formula [33, 50]:

$$
L^{\dagger}=\left[L+(N+M)^{-1} e e^{T}\right]^{-1}-(N+M)^{-1} e e^{T} .
$$

The speed up in this case is however in terms of the absolute run time. The overall complexity is still $O\left((N+M)^{3}\right)$, but with smaller constants. We note that it is also possible to speed up the inversion by exploiting the bipartite nature of $\Gamma$ [33], although we have not used this in our implementation.

The complexity of the eigenvector computation is $O\left(M^{3} d^{3}\right)$, while that of the orthogonal rounding is $O\left(M d^{3}\right)$. The total complexity of GRET-SPEC, say, using a linear-time Laplacian inversion, is (up to logarithmic factors)

$$
O\left(|E(\Gamma)|+(M d)^{3}\right) .
$$

The main computational blocks in GRET-SDP are identical to that in GRET-SPEC, plus the SDP computation. The SDP solution can be computed in polynomial 
time using interior-point programming [67]. In particular, the complexity of computing an $\varepsilon$-accurate solution using interior-point solvers such as SDPT3 [58] is $O\left((M d)^{4.5} \log (1 / \varepsilon)\right)$. It is possible to lower this complexity by exploiting the particular structure of $\left(\mathrm{P}_{2}\right)$. For example, notice that the constraint matrices in $\left(\mathrm{P}_{2}\right)$ have at most one non-zero coefficient. Using the algorithm in [30], one can then bring down the complexity of the SDP to $O\left((M d)^{3.5} \log (1 / \varepsilon)\right)$. By considering a penalized version of the SDP, we can use first-order solvers such as TFOCS [5] to further cut down the dependence on $M$ and $d$ to $O\left((M d)^{3} \varepsilon^{-1}\right)$, but at the cost of a stronger dependence on the accuracy. The quest for efficient SDP solvers is currently an active area of research. Fast SDP solvers have been proposed that exploit either the low-rank structure of the SDP solution [11, 38] or the simple form of the linearity constraints in $\left(\mathrm{P}_{2}\right)$ [64]. More recently, a sublinear time approximation algorithm for SDP was proposed in [22]. The complexity of GRET-SDP using a linear-time Laplacian inversion and an interior-point SDP solver is thus

$$
O\left(|E(\Gamma)|+(M d)^{4.5} \log (1 / \varepsilon)+(M d)^{3}\right) .
$$

For problems where the size of the SDP variable is within 150, we can solve $\left(\mathrm{P}_{2}\right)$ in reasonable time on a standard PC using SDPT3 [58] or CVX [29]. We use CVX for the numerical experiments in Section 6 that involve small-to-moderate sized SDP variables. For larger SDP variables, one can use the low-rank structure of $\left(\mathrm{P}_{2}\right)$ to speed up the computation. In particular, we were able to solve for SDP variables of size up to $2000 \times 2000$ using SDP LR [11] that exploits this low-rank structure .

\section{EXACT RECOVERY}

We now examine conditions on the membership graph under which the proposed spectral and convex relaxations can recover the global coordinates from the knowledge of the clean local coordinates (and the membership graph). More precisely, let $\bar{x}_{1}, \ldots, \bar{x}_{N}$ be the true coordinates of a point cloud in $\mathbb{R}^{d}$. Suppose that the point cloud is divided into patches whose membership graph is $\Gamma$, and that we are provided the measurements

$$
x_{k, i}=\bar{O}_{i}^{T}\left(\bar{x}_{k}-\bar{t}_{i}\right) \quad(k, i) \in E(\Gamma),
$$

for some $\bar{O}_{i} \in \mathbb{O}(d)$ and $\bar{t}_{i} \in \mathbb{R}^{d}$. The patch-stress matrix $C$ is constructed from $\Gamma$ and the clean measurements (25). The question is under what conditions on $\Gamma$ can $\bar{x}_{1}, \ldots, \bar{x}_{N}$ be recovered by our algorithm? We will refer to this as exact recovery.

To express exact recovery in the matrix notation introduced earlier, define

$$
\bar{Z}=\left[\bar{x}_{1} \cdots \bar{x}_{N} \bar{t}_{1} \cdots \bar{t}_{M}\right] \in \mathbb{R}^{d \times(N+M)},
$$

and

$$
\bar{O}=\left[\bar{O}_{1} \cdots \bar{O}_{M}\right] \in \mathbb{R}^{d \times M d} .
$$

Then, exact recovery means that for some $\Omega \in \mathbb{O}(d)$ and $t \in \mathbb{R}^{d}$,

$$
Z^{\star}=\Omega \bar{Z}+t e^{T} \text {. }
$$

Henceforth, we will always assume that $\Gamma$ is connected (clearly one cannot have exact recovery otherwise).

Conditions for exact recovery have previously been examined by Zha and Zhang [68] in the context of tangent-space alignment in manifold learning, and later by Gortler et al. [25] from the perspective of rigidity theory. In particular, they show 
that the so-called notion of affine rigidity is sufficient for exact recovery using the spectral method. Moreover, the authors in [68, 25] relate this notion of rigidity to other standard notions of rigidity, and provide conditions on a certain hypergraph constructed from the patch system that can guarantee affine rigidity. The purpose of this section is to briefly introduce the rigidity results in [68, 25] and relate these to the properties of the membership graph $\Gamma$ (and the patch-stress matrix $C$ ). We note that the authors in [68, 25] directly examine the uniqueness of the global coordinates, while we are concerned with the uniqueness of the patch transforms obtained by solving $\left(\mathrm{P}_{1}\right)$ and $\left(\mathrm{P}_{2}\right)$. The uniqueness of the global coordinates is then immediate:

Proposition 3 (Uniqueness and Exact Recovery). If $\left(\mathrm{P}_{1}\right)$ and $\left(\mathrm{P}_{2}\right)$ have unique solutions, then 26) holds for both GRET-SPEC and GRET-SDP.

At this point, we note that if a patch has less than $d+1$ points, then even when $\bar{x}_{1}, \ldots, \bar{x}_{N}$ are the unique set of coordinates that satisfy 25, we cannot guarantee $\bar{O}_{1}, \ldots, \bar{O}_{M}$ and $\bar{t}_{1}, \ldots, \bar{t}_{M}$ to be unique. Therefore, we will work under the mild assumption that each patch has at least $d+1$ non-degenerate points, so that the patch transforms are uniquely determined from the global coordinates.

We now formally define the notion of affine rigidity. Although phrased differently, it is in fact identical to the definitions in [68, 25]. Henceforth, by affine transform, we will mean the group of non-singular affine maps on $\mathbb{R}^{d}$. Affine rigidity is a property of the patch-graph $\Gamma$ and the local coordinates $\left(x_{k, i}\right)$. In keeping with [25], we will together call these the patch framework and denote it by $\Theta=\left(\Gamma,\left(x_{k, i}\right)\right)$.

Definition 4 (Affine Rigidity). Let $y_{1}, \ldots, y_{N} \in \mathbb{R}^{d}$ be such that, for affine transforms $A_{1}, \ldots, A_{M}$,

$$
y_{k}=A_{i}\left(x_{k, i}\right) \quad(k, i) \in E(\Gamma) .
$$

The patch framework $\Theta=\left(\Gamma,\left(x_{k, i}\right)\right)$ is affinely rigid if $y_{1}, \ldots, y_{N}$ is identical to $\bar{x}_{1}, \ldots \bar{x}_{N}$ up to a global affine transform.

Since each patch has $d+1$ points, we now give a characterization of affine rigidity that will be useful later on.

Proposition 5. A patch framework $\Theta=\left(\Gamma,\left(x_{k, i}\right)\right)$ is affinely rigid if and only if for any $F \in R^{d \times M d}$ such that $\operatorname{Tr}\left(C F^{T} F\right)=0$ we must have $F=A \bar{O}$ for some non-singular $A \in R^{d \times d}$.

Before proceeding to the proof, note that $\bar{O}$ and $\bar{G}=\bar{O}^{T} \bar{O}$ are solutions of $\left(\mathrm{P}_{1}\right)$ and $\left(\mathrm{P}_{2}\right)$ (this was the basis of Proposition 3), and the objective in either case is zero. Indeed, from (25), we can write $\bar{Z} L=\overline{O B}$. Since $\Gamma$ is connected,

$$
\bar{Z}=\bar{O} B L^{\dagger}+t e^{T} \quad\left(t \in \mathbb{R}^{d}\right) .
$$

Using (27), it is not difficult to verify that $\phi(\bar{Z}, \bar{O})=\operatorname{Tr}(C \bar{G})$. Moreover, it follows from (25) that $\phi(\bar{Z}, \bar{O})=0$. Therefore,

$$
\operatorname{Tr}(C \bar{G})=\operatorname{Tr}\left(C \bar{O}^{T} \bar{O}\right)=0 .
$$

Using an identical line of reasoning, we also record another fact. Let $F=\left[F_{1}, \ldots, F_{M}\right]$ where each $F_{i} \in \mathbb{R}^{d \times d}$. Suppose there exists $y_{1}, \ldots, y_{N} \in \mathbb{R}^{d}$ and $t_{1}, \ldots, t_{M} \in \mathbb{R}^{d}$ such that

$$
y_{k}=F_{i} x_{k, i}+t_{i} \quad(k, i) \in E(\Gamma) .
$$




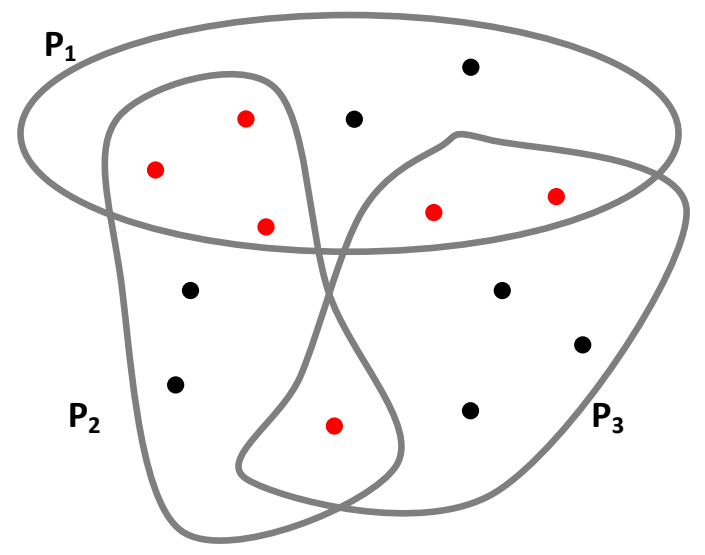

FIGURE 2. Instance of three overlapping patches, where the overlapping points are shown in red. In this case, $P_{3}$ cannot be registered with either $P_{1}$ or $P_{2}$ due to insufficient overlap. Therefore, the patches cannot be localized in two dimension using, for example, [69, 17] that work by registering pairs of patches. The patches can however be registered using GRET-SPEC and GRET-SDP since the ordered patches $P_{1}, P_{2}, P_{3}$ form a graph lateration in $\mathbb{R}^{2}$.

Then $Y=\left[y_{1}, \ldots, y_{N}, t_{1}, \ldots, t_{M}\right] \in \mathbb{R}^{d \times(N+M)}$ satisfies

$$
Y=F B L^{\dagger}+t e^{T}
$$

and $\operatorname{Tr}\left(C F^{T} F\right)=0$.

of Proposition 5 For any $F$ such that $\operatorname{Tr}\left(C F^{T} F\right)=0$, letling

$$
\left[y_{1}, \ldots, y_{N}, t_{1}, \ldots, t_{M}\right]=F B L^{\dagger},
$$

we have (29). By the affine rigidity assumption, we must then have $y_{k}=A \bar{x}_{k}+t$ for some non-singular $A \in \mathbb{R}^{d \times d}$ and $t \in \mathbb{R}^{d}$. Since each patch contains $d+1$ non-degenerate points, it follows that $F=A \bar{O}$.

In the other direction, assume that $y_{1}, \ldots, y_{N} \in \mathbb{R}^{d}$ satisfy (29). We know that $\operatorname{Tr}\left(C F^{T} F\right)=0$ and hence $F=A \bar{O}$ for some non-singular $A$. Using (30), we immediately have $y_{k}=A \bar{x}_{k}+t$.

Note that $\operatorname{Tr}\left(C F^{T} F\right)=0$ implies that the rows of $F$ are in the null space of $C$. Therefore, the combined facts that $\operatorname{Tr}\left(C F^{T} F\right)=0$ and $F=A \bar{O}$ for some nonsingular $A \in R^{d \times d}$ is equivalent to saying that null space of $C$ is within the row span of $\bar{O}$. The following result then follows as a consequence of (5).

Corollary 6. A patch framework $\Theta=\left(\Gamma,\left(x_{k, i}\right)\right)$ is affinely rigid if and only if the rank of $C$ is $(M-1) d$.

The corollary gives an easy way to check for affine rigidity. However, it is not clear what construction of $\Gamma$ will ensure such property. In [68], the notion of graph 
lateration was introduced that guarantees affine rigidity. Namely, $\Gamma$ is said to be a graph lateration (or simply laterated) if there exists an reordering of the patch indices such that, for every $i \geq 2, P_{i}$ and $P_{1} \cup \cdots \cup P_{i-1}$ have at least $d+1$ nondegenerate nodes in common. An example of a graph lateration is shown in Figure 2.

Theorem 7 ([68]). If $\Gamma$ is laterated and the local coordinates are non-degenerate then the framework $\Theta$ is affinely rigid.

Next, we turn to the exact recovery conditions for $\left(\mathrm{P}_{2}\right)$. The appropriate notion of rigidity in this case is that of universal rigidity [27]. Just as we defined affine rigidity earlier, we can phrase universal rigidity as follows.

Definition 8 (Universal Rigidity). Suppose that (25) holds. Let $x_{1}, \ldots, x_{N} \in \mathbb{R}^{s}(s \geq d)$ be such that, for some orthogonal $O_{i} \in \mathbb{R}^{s \times d}$ and $t_{i} \in \mathbb{R}^{s}$,

$$
x_{k}=O_{i} x_{k, i}+t_{i} \quad(k, i) \in E .
$$

We say that the patch framework $\Theta=\left(\Gamma,\left(x_{k, i}\right)\right)$ is universally rigid in $\mathbb{R}^{d}$ if for any such $\left(x_{k}\right)$, we have $x_{k}=\Omega \bar{x}_{k}$ for some orthogonal $\Omega \in \mathbb{R}^{s \times d}$.

By orthogonal $\Omega$, we mean that the columns of $\Omega$ are orthogonal and of unit norm (i.e., $\Omega$ can be seen an orthogonal transform in $\mathbb{R}^{s}$ by identifying $\mathbb{R}^{d}$ as a subspace of $\mathbb{R}^{s}$ ).

Following exactly the same arguments used to establish Proposition 5 , one can derive the following.

Proposition 9. The following statements are equivalent:

(a) A patch framework $\Theta=\left(\Gamma,\left(x_{k, i}\right)\right)$ is universally rigid in $\mathbb{R}^{d}$.

(b) Let $O \in \mathbb{R}^{s \times M d}(s \geq d)$ be such that $O_{i}^{T} O_{i}=I_{d}$ for all $i$. Then

$$
\operatorname{Tr}\left(C O^{T} O\right)=0 \Rightarrow O=\Omega \bar{O} \text { for some orthogonal } \Omega \in \mathbb{R}^{s \times d} .
$$

The question then is under what conditions is the patch framework universally rigid? This was also addressed in [25] using a graph construction derived from $\Gamma$ called the body graph. This is given by $\Gamma_{B}=\left(V_{B}, E_{B}\right)$, where $V_{B}=\{1,2, \ldots, N\}$ and $(k, l) \in E_{B}$ if and only if $x_{k}$ and $x_{l}$ belong to the same patch (cf. Figure 3 ). Next, the following distances are associated with $\Gamma_{B}$ :

$$
d_{k l}=\left\|x_{k, i}-x_{l, i}\right\| \quad(k, l) \in E_{B},
$$

where $x_{k}, x_{l} \in P_{i}$, say. Note that the above assignment is independent of the choice of patch. A set of points $\left(x_{k}\right)_{k \in V}$ in $\mathbb{R}^{s}$ is said to be a realization of $\left\{d_{k l}:(k, l) \in E\right\}$ in $\mathbb{R}^{s}$ if $d_{k l}=\left\|x_{k}-x_{l}\right\|$ for $(k, l) \in E$.

It was shown in [25] that $\Theta=\left(\Gamma,\left(x_{k, i}\right)\right)$ is universally rigid if and only if $\Gamma_{B}$ with distances $\left\{d_{k l}:(k, l) \in E\right\}$ has a unique realization in $\mathbb{R}^{s}$ for all $s \geq d$. Moreover, in such situation, using the distances as the constraints, an SDP relaxation was proposed in [56, 71] for finding the unique realization. We note that although the SDP in [56] has the same condition for exact recovery as $\left(\mathrm{P}_{2}\right)$, it is computationally more demanding than $\left(\mathrm{P}_{2}\right)$ since the number of variables is $O\left(N^{2}\right)$ for this SDP, instead of $O\left(M^{2}\right)$ as in $\left(\mathrm{P}_{2}\right)$ (for most applications, $\left.M \ll N\right)$. Moreover, as we will see shortly in Section 6. $\left(\mathrm{P}_{2}\right)$ also enjoys some stability properties which has not been established for the SDP in [56]. 


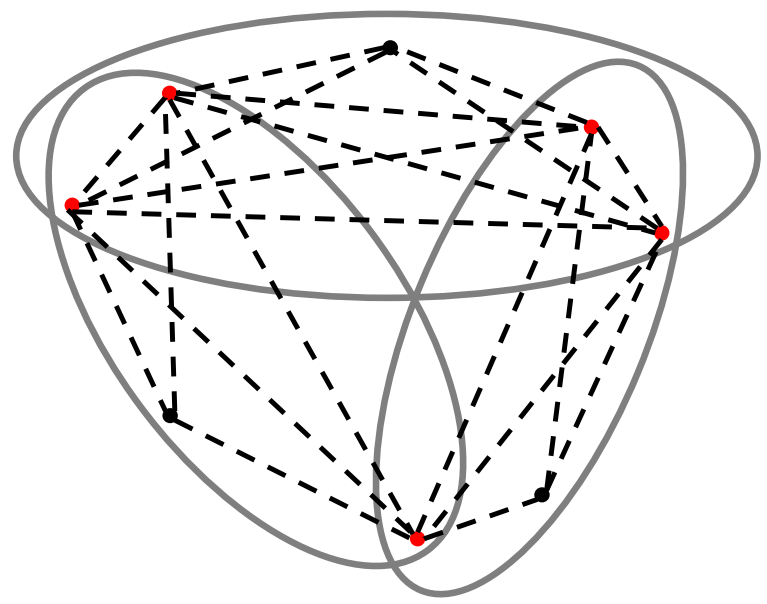

FIGURE 3. This shows the body graph for a 3-patch system (patches marked with ovals, points marked with dots). The edges of the body graph are obtained by connecting points that belong to the same patch. The edges within a given patch are marked with the same color. GRET-SDP can successfully register all the patches if the body graph is rigid in a certain sense.

Finally, we note that universal rigidity is a weaker condition on $\Gamma$ than affine rigidity.

Theorem 10 ([56], Theorem 2). If a patch framework is affinely rigid, then it is universally rigid.

In [25], it was also shown that the reverse implication is not true using an counterexample for which the patch framework fails to be affinely rigid, but for which the body graph (a Cauchy polygon) has an unique realization in any dimension [15]. This means that GRET-SDP can solve a bigger class of problems than GRET-SPEC, which is perhaps not surprising.

\section{RANDOMIZED RANK TEST}

Corollary 6 tells us by checking the rank of the patch stress matrix $C$, we can tell whether a patch framework is affinely rigid. In this regard, the patch-stress matrix serves the same purpose as the so-called alignment matrix in [68] and the affinity matrix in [25]. The only difference is that the kernel of $C$ represents the degree of freedom of the affine transform, whereas kernel of alignment or affinity matrix directly tell us the degree of freedom of the point coordinates. As suggested in [25], an efficient randomized test for affine rigidity using the concept of affinity matrix can be easily derived. In this section, we describe a randomized test based on patch stress matrix, which parallels the proposal in [25]. This procedure is also similar in spirit to the randomized tests for generic local rigidity by Hendrickson [31], for 
generic global rigidity by Gortler et al. [26], and for matrix completion by Singer and Cucuringu [54].

Let us continue to denote the patch-stress matrix obtained from $\Gamma$ and the measurements (25) by $C$. We will use $C_{0}$ to denote the patch-stress matrix obtained from the same graph $\Gamma$, but using the (unknown) original coordinates as measurements, namely,

$$
x_{k, i}=\bar{x}_{k} \quad(k, i) \in \Gamma .
$$

The advantage of working with $C_{0}$ over $C$ is that the former can be computed using just the global coordinates, while the latter requires the knowledge of the global coordinates as well as the clean transforms. In particular, this only requires us to simulate the global coordinates. Since the coordinates of points in a given patch are determined up to a rigid transform, we claim the following (cf. Section 8.1 for a proof).

Proposition 11 (Rank equivalence). For a fixed $\Gamma, C$ and $C_{0}$ have the same rank.

In other words, the rank of $C_{0}$ can be used to certify exact recovery. The proposed test is based on Proposition 8.1. and the fact that if two different generic configurations are used as input in (32) (for the same $\Gamma$ ), then the patch-stress matrices they produce would have the same rank. By generic, we mean that the coordinates of the configuration do not satisfy any non-trivial algebraic equation with rational coefficients [26].

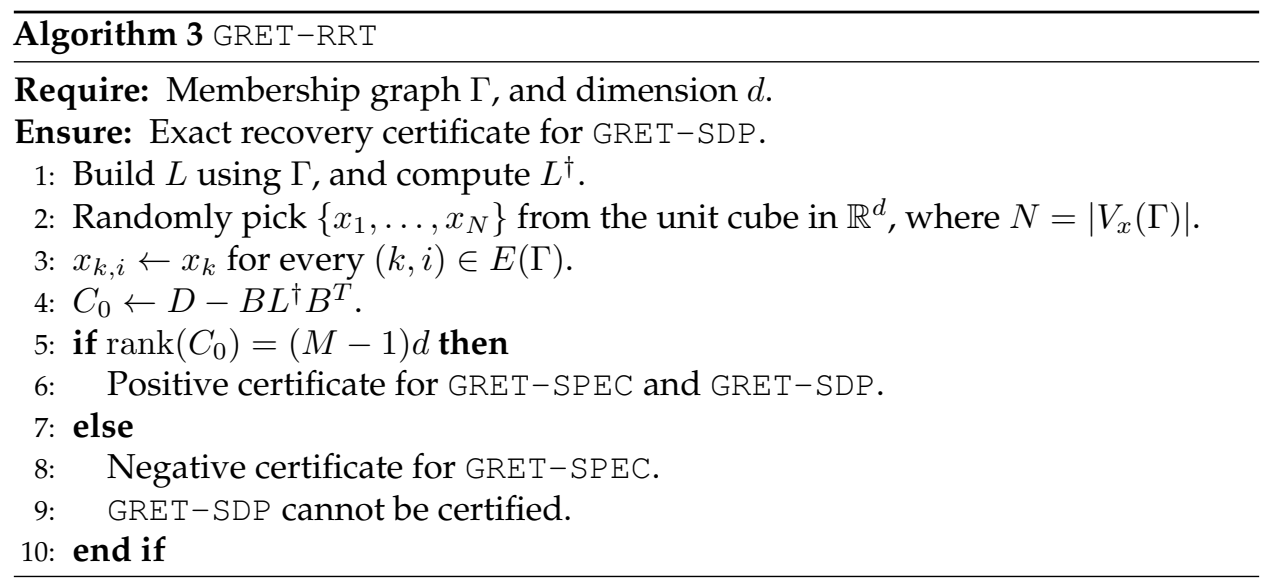

The complete test called "GRET-Randomized Rank Test" (GRET-RRT) is described in Algorithm 3 . Note that the main computations in GRET-RRT are the Laplacian inversion (which is also required for the registration algorithm) and the rank computation.

\section{StABility ANALYSis}

We have so far studied the problem of exact recovery from noiseless measurements. In practice, however, the measurements are invariably noisy. This brings us to the question of stability, namely how stable are GRET-SPEC and GRET-SDP to perturbations in the measurements? Numerical results (to be presented in the next 
Section) show that both the spectral and semidefinite relaxations are quite stable to perturbations. In particular, the reconstruction error degrades quite gracefully with the increase in noise (reconstruction error is the gap between the outputs with clean and noisy measurements). In this Section, we try to quantify these empirical observations. In particular, we prove that, for a specific noise model, the reconstruction error grows at most linearly with the level of noise for the semidefinite relaxation.

The noise model we consider is the "bounded" noise model. Namely, we assume that the measurements are obtained through bounded perturbations of the clean measurements in (25). More precisely, we suppose that we have a membership graph $\Gamma$, and that the observed local coordinates are of the form

$$
x_{k, i}=\bar{O}_{i}^{T}\left(\bar{x}_{k}-\bar{t}_{i}\right)+\epsilon_{k, i}, \quad\left\|\epsilon_{k, i}\right\| \leq \varepsilon \quad(k, i) \in E(\Gamma) .
$$

In other words, every coordinate measurement is offset within a ball of radius $\varepsilon$ around the clean measurements. Here, $\varepsilon$ is a measure of the noise level per measurement. In particular, $\varepsilon=0$ corresponds to the case where we have the clean measurements 25.

Since the coordinates of points in a given patch are determined up to a rigid transform, it is clear that the above problem is equivalent to the one where the measurements are

$$
x_{k, i}=\bar{x}_{k}+\epsilon_{k, i}, \quad\left\|\epsilon_{k, i}\right\| \leq \varepsilon \quad(k, i) \in E(\Gamma) .
$$

By equivalent, we mean that the reconstruction errors obtained using either 33 or (34) are equal. The reason we use the latter measurements is that the analysis in this case is much more simple.

The reconstruction error is defined as follows. Generally, let $Z^{\star}$ be the output of Algorithms 1 and 2 using (34) as input, and let

$$
Z_{0} \stackrel{\text { def }}{=}\left[\bar{x}_{1} \cdots \bar{x}_{N} 0 \cdots 0\right] \in \mathbb{R}^{d \times(N+M)},
$$

where we assume that the centroid of $\left\{\bar{x}_{1}, \cdots, \bar{x}_{N}\right\}$ is at the origin.

Ideally, we would require that $Z^{\star}=Z_{0}$ (up to a rigid transformation) when there is no noise, that is, when $\varepsilon=0$. This is the exact recovery phenomena that we considered earlier. In general, the gap between $Z_{0}$ and $Z^{\star}$ is a measure of the reconstruction quality. Therefore, we define the reconstruction error to be

$$
\eta=\min _{\Theta \in \mathbb{O}(d)}\left\|Z^{\star}-\Theta Z_{0}\right\|_{F} .
$$

Note that we are not required to factor out the translation since $Z_{0}$ is centered by construction.

Our main results are the following.

Theorem 12 (Stability of GRET-SPEC). Assume that $R$ is the radius of the smallest Euclidean ball that encloses the clean configuration $\left\{\bar{x}_{1}, \ldots, \bar{x}_{N}\right\}$. For fixed noise level $\varepsilon \geq$ 0 and membership graph $\Gamma$, suppose we input the noisy measurements (34) to GRET-SPEC. If $\operatorname{rank}\left(C_{0}\right)=(M-1) d$, then we have the following bound for GRET-SPEC:

$$
\eta \leq \frac{|E(\Gamma)|^{1 / 2}}{\lambda_{2}(L)}\left(K_{1} \varepsilon+K_{2} \varepsilon^{2}\right),
$$


where

$K_{1}=\frac{8 \pi R}{\mu_{d+1}(C)} \sqrt{2 M N|E(\Gamma)|(2+N) d(d+1)}\left(4 R \frac{\sqrt{N|E(\Gamma)|}}{\lambda_{2}(L)}+1\right)+\sqrt{2+N+M}$.

and

$$
K_{2}=\frac{8 \pi R}{\mu_{d+1}(C)} \sqrt{2 M N|E(\Gamma)|(2+N) d(d+1)}\left(2 \frac{\sqrt{N|E(\Gamma)|}}{\lambda_{2}(L)}+1\right) .
$$

Here $\lambda_{2}(L)$ is the second smallest eigenvalue of $L$.

We assume here that $\mu_{d+1}(C)$ is non-zerd ${ }^{3}$ The bounds here are in fact quite loose. Note that when $\varepsilon=0$, we recover the exact recovery result for GRET-SPEC provided in [68, 25].

Theorem 13 (Stability of GRET-SDP). Under the conditions of Theorem 12 , we have the following for GRET-SDP:

$$
\eta \leq \frac{|E(\Gamma)|^{1 / 2}}{\lambda_{2}(L)}\left[32 \sqrt{2 d(d+1)(2+N)|E(\Gamma)|} \mu_{d+1}^{-1 / 2}\left(C_{0}\right) R+\sqrt{2+N+M}\right] \varepsilon .
$$

The bounds are again quite loose. The main point here is that the reconstruction error for GRET-SDP is within a constant factor of the noise level. In particular, Theorem 13 subsumes the exact recovery condition $\operatorname{rank}\left(C_{0}\right)=(M-1) d$ described in Section 3 .

The rest of this Section is devoted to the proofs of Theorem 12 and 13 First, we introduce some notations.

Notations. Note that the patch-stress matrix in $\left(\mathrm{P}_{1}\right)$ is computed from the noisy measurements (34), and the same patch-stress matrix is used in $\left(\mathrm{P}_{2}\right)$. The quantities $G^{\star}, W^{\star}, O^{\star}$, and $Z^{\star}$ are as defined in Algorithms 1 and 2 . We continue to denote the clean patch-stress matrix by $C_{0}$. Define

$$
O_{0} \stackrel{\text { def }}{=}\left[I_{d} \cdots I_{d}\right] \text { and } G_{0} \stackrel{\text { def }}{=} O_{0}^{T} O_{0} .
$$

Let $e_{1}, \ldots, e_{d}$ be the standard basis vectors of $\mathbb{R}^{d}$, and let $e$ be the all-ones vector of length $M$. Define

$$
s_{i} \stackrel{\text { def }}{=} \frac{1}{\sqrt{M}} e \otimes e_{i} \in \mathbb{R}^{M d} \quad(1 \leq i \leq d) .
$$

Note that every $d \times d$ block of $G_{0}$ is $I_{d}$, and that we can write

$$
G_{0}=\sum_{i=1}^{d} M s_{i} s_{i}^{T}
$$

We first present an estimate that applies generally to both algorithms. The proof is provided in Section 8.2 .

\footnotetext{
${ }^{3}$ Numerical experiments suggest that this is indeed the case if $\operatorname{rank}\left(C_{0}\right)=(M-1) d$. In fact, we notice a growth in the eigenvalue with the increase in noise level. We have however not been able to prove this fact.
} 
Proposition 14 (Basic estimate). Let $R$ be the radius of the smallest Euclidean ball that encloses the clean configuration. Then, for any arbitrary $\Theta$,

$$
\left\|Z^{\star}-\Theta Z_{0}\right\|_{F} \leq \frac{|E(\Gamma)|^{1 / 2}}{\lambda_{2}(L)}\left[R(2+N)^{1 / 2}\left\|O^{\star}-\Theta O_{0}\right\|_{F}+\varepsilon(2+N+M)^{1 / 2}\right] .
$$

In other words, the reconstruction error in either case is controlled by the rounding error:

$$
\delta=\min _{\Theta \in \mathbb{O}(d)}\left\|O^{\star}-\Theta O_{0}\right\|_{F} .
$$

The rest of this Section is devoted to obtaining a bound on $\delta$ for GRET-SPEC and GRET-SDP. In particular, we will show that $\delta$ is of the order of $\varepsilon$ in either case. Note that the key difference between the two algorithms arises from the eigenvector rounding, namely the assignment of the "unrounded" orthogonal transform $W^{\star}$ (respectively from the patch-stress matrix and the optimal Gram matrix). The analysis in going from $W^{\star}$ to the rounded orthogonal transform $O^{\star}$, and subsequently to $Z^{\star}$, is however common to both algorithms.

We now bound the error in (39) for both algorithms. Note that we can generally write

$$
W^{\star}=\left[\sqrt{\alpha_{1}} u_{1} \cdots \sqrt{\alpha_{d}} u_{d}\right]^{T}
$$

where $u_{1}, \ldots, u_{d}$ are orthonormal. In GRET-SPEC, each $\alpha_{i}=M$, while in GRET-SDP we set $\alpha_{i}$ using the eigenvalues of $G^{\star}$.

Our first result gives a control on the quantities obtained using eigenvector rounding in terms of their Gram matrices.

Lemma 15 (Eigenvector rounding). There exist $\Theta \in \mathbb{O}(d)$ such that

$$
\left\|W^{\star}-\Theta O_{0}\right\|_{F} \leq \frac{4}{\sqrt{M}}\left\|W^{\star T} W^{\star}-G_{0}\right\|_{F} .
$$

Next, we use a result by $\mathrm{Li}$ [42] to get a bound on the error after orthogonal rounding.

Lemma 16 (Orthogonal rounding). For arbitrary $\Theta \in \mathbb{O}(d)$,

$$
\left\|O^{\star}-\Theta O_{0}\right\|_{F} \leq 2 \sqrt{d+1}\left\|W^{\star}-\Theta O_{0}\right\|_{F} .
$$

The proofs of Lemma 15 and 16 are provided in Appendices 8.3 and 8.4 At this point, we record a result from [44] which is repeatedly used in the proof of these lemmas and elsewhere.

Lemma 17 (Mirsky, [44]). Let $\|\cdot\| \mid$ be some unitarily invariant norm, and let $A, B \in$ $\mathbb{R}^{n \times n}$. Then

$$
\left\|\operatorname{diag}\left(\sigma_{1}(A)-\sigma_{1}(B), \cdots, \sigma_{n}(A)-\sigma_{n}(B)\right)\right\| \leq\|A-B\| .
$$

In particular, the above result holds for the Frobenius and spectral norms.

By combining Lemma 15 and 16 , we have the following bound for (39):

$$
\delta \leq 8 \sqrt{\frac{d+1}{M}}\left\|W^{\star T} W^{\star}-G_{0}\right\|_{F} .
$$

We now bound the quantity on the right in (40) for GRET-SPEC and GRET-SDP. 
5.1. Bound for GRET-SPEC. For the spectral relaxation, this can be done using the Davis-Kahan theorem [7]. Note that from (18), we can write

$$
\frac{1}{M}\left(W^{\star T} W^{\star}-G_{0}\right)=\sum_{i=1}^{d} r_{i} r_{i}^{T}-\sum_{j=1}^{d} s_{j} s_{j}^{T} .
$$

Following [7, Ch. 7], let $A$ be some symmetric matrix and $S$ be some subset of the real line. Denote $P_{A}(S)$ to be the orthogonal projection onto the subspace spanned by the eigenvectors of $A$ whose eigenvalues are in $S$. A particular implication of the Davis-Kahan theorem is that

$$
\left\|P_{A}\left(S_{1}\right)-P_{B}\left(S_{2}\right)\right\|_{\mathrm{sp}} \leq \frac{\pi}{2 \rho\left(S_{1}^{c}, S_{2}\right)}\|A-B\|_{\mathrm{sp}},
$$

where $S_{1}^{c}$ is the complement of $S_{1}$, and $\rho\left(S_{1}, S_{2}\right)=\min \left\{|u-v|: u \in S_{1}, v \in S_{2}\right\}$.

In order to apply (42) to (41), set $A=C, B=C_{0}, S_{1}=\left[\mu_{1}(C), \mu_{d}(C)\right]$, and $S_{2}=\{0\}$. If $\operatorname{rank}\left(C_{0}\right)=(M-1) d$, then $P_{B}\left(S_{2}\right)=\sum_{j=1}^{d} s_{j} s_{j}^{T}$. Applying (42), we get

$$
\left\|W^{\star T} W^{\star}-G_{0}\right\|_{\mathrm{sp}} \leq \frac{M \pi}{2 \mu_{d+1}(C)}\left\|C-C_{0}\right\|_{F} .
$$

Now, it is not difficult to verify that for the noise model (34),

$$
\left\|C-C_{0}\right\|_{F} \leq 2 \sqrt{N|E(\Gamma)|}\left[\left(4 R \frac{\sqrt{N|E(\Gamma)|}}{\lambda_{2}(L)}+1\right) \varepsilon+\left(2 \frac{\sqrt{N|E(\Gamma)|}}{\lambda_{2}(L)}+1\right) \varepsilon^{2}\right] .
$$

Combining Proposition 14 with (40), (43), and (44), we arrive at Theorem 12 .

5.2. Bound for GRET-SDP. To analyze the bound for GRET-SDP, we require further notations. Recall (36), and let $S$ be the space spanned by $\left\{s_{1}, \ldots, s_{d}\right\} \subset \mathbb{R}^{M d}$, and let $\bar{S}$ be the orthogonal complement of $S$ in $\mathbb{R}^{M d}$. In the sequel, we will be required to use matrix spaces arising from tensor products of vector spaces. In particular, given two subspaces $U$ and $V$ of $\mathbb{R}^{M d}$, denote by $U \otimes V$ the space spanned by the rank-one matrices $\left\{u v^{T}: u \in U, v \in V\right\}$. In particular, note that $G_{0}$ is in $S \otimes S$.

Let $A \in \mathbb{R}^{M d \times M d}$ be some arbitrary matrix. We can decompose it into

$$
A=P+Q+T
$$

where

$$
P \in S \otimes S, Q \in(S \otimes \bar{S}) \cup(\bar{S} \otimes S), \text { and } T \in \bar{S} \otimes \bar{S} .
$$

We record a result about this decomposition from Wang and Singer [63].

Lemma 18 ([63], pg. 7). Suppose $G_{0}+\Delta \succeq 0$ and $\Delta_{i i}=0(1 \leq i \leq M)$. Let $\Delta=P+Q+T$ as in (45). Then

$$
T \succeq 0, \quad \text { and } \quad P_{i j}=-\frac{1}{M} \sum_{l=1}^{M} T_{l l} \quad(1 \leq i, j \leq M) .
$$

It is not difficult to verify that $\operatorname{Tr}\left(C_{0} G_{0}\right)=0$ and that $C_{0} \succeq 0$. From (37), we have

$$
0=\operatorname{Tr}\left(C_{0} G_{0}\right)=\sum_{i=1}^{d} s_{i}^{T} C_{0} s_{i} \geq 0 .
$$


Since each term in the above sum is non-negative, $C_{0} s_{i}=0$ for $1 \leq i \leq d$. In other words, $S$ is contained in the null space of $C_{0}$. Moreover, if $\operatorname{rank}\left(C_{0}\right)=(M-1) d$, then $S$ is exactly the null space of $C_{0}$. Based on this observation, we give a bound on the residual $T$.

Proposition 19 (Bound on the residual). Suppose that $\operatorname{rank}\left(C_{0}\right)=(M-1) d$. Decompose $\Delta=P+Q+T$ as in 45 . Then

$$
\operatorname{Tr}(T) \leq 4 \mu_{d+1}^{-1}\left(C_{0}\right)|E(\Gamma)| \varepsilon^{2} .
$$

Proof. The main idea here is to compare the objective in $\left(\mathrm{P}_{0}\right)$ with the trace of $T$. To do so, we introduce the following notations. Let $\lambda_{1}, \cdots, \lambda_{M d}$ be the full set of eigenvalues of $G^{\star}$ sorted in non-increasing order, and $q_{1}, \ldots, q_{M d}$ be the corresponding eigenvectors. Define

$$
O^{\star \star} \stackrel{\text { def }}{=}\left[\begin{array}{lll}
\sqrt{\lambda_{1}} & \cdots \sqrt{\lambda_{M d}} q_{M d}
\end{array}\right]^{T} \in \mathbb{R}^{M d \times M d},
$$

and $O_{i}^{\star \star}$ to be the $i$-th $M d \times d$ block of $O^{\star \star}$, that is, $O^{\star \star} \stackrel{\text { def }}{=}\left[O_{1}^{\star \star} \cdots O_{M}^{\star \star}\right]$.

By construction, $G^{\star}=O^{\star \star T} O^{\star \star}$. Moreover, by feasibility,

$$
G_{i i}^{\star}=O_{i}^{\star \star T} O_{i}^{\star \star}=I_{d} \quad(1 \leq i \leq M) .
$$

Thus the $d$ columns of $O_{i}^{\star \star}$ form an orthonormal system in $\mathbb{R}^{M d}$. Now define

$$
Z^{\star \star} \stackrel{\text { def }}{=} O^{\star \star} B L^{\dagger} \in \mathbb{R}^{M d \times(N+M)} .
$$

In particular, we will use the fact that $\left(Z^{\star \star}, O^{\star \star}\right)$ are the minimizers of the unconstrained program

$$
\min _{(Z, O)} \sum_{(k, i) \in E(\Gamma)}\left\|Z e_{k i}-O_{i} x_{k, i}\right\|^{2} \quad \text { s.t. } \quad Z \in \mathbb{R}^{M d \times(N+M)}, O \in \mathbb{R}^{M d \times M d} .
$$

Note that $\operatorname{Tr}\left(C_{0} G^{\star}\right)=\operatorname{Tr}\left(C_{0}\left(G_{0}+\Delta\right)\right)=\operatorname{Tr}\left(C_{0} T\right)$. Now, by Lemma (18), $T \succeq 0$. Therefore, writing

$$
T=\sum_{i} v_{i} v_{i}^{T} \quad\left(v_{i} \in \bar{S}\right)
$$

we get

$$
\operatorname{Tr}\left(C_{0} T\right)=\sum_{i} v_{i}^{T} C_{0} v_{i} \geq \mu_{d+1}\left(C_{0}\right) \sum_{i} v_{i}^{T} v_{i}=\mu_{d+1}\left(C_{0}\right) \operatorname{Tr}(T) .
$$

Therefore,

$$
\operatorname{Tr}(T) \leq \mu_{d+1}^{-1}\left(C_{0}\right) \operatorname{Tr}\left(C_{0} G^{\star}\right) .
$$

We are done if we can bound the term on the right. To do so, we note from 477 that

$$
\operatorname{Tr}\left(C_{0} G^{\star}\right)=\operatorname{Tr}\left(C_{0} O^{\star \star T} O^{\star \star}\right)=\min _{Z \in \mathbb{R}^{M d \times N+M}} \sum_{(k, i) \in E(\Gamma)}\left\|Z e_{k i}-O_{i}^{\star \star} \bar{x}_{k}\right\|^{2} .
$$

Therefore,

$$
\operatorname{Tr}\left(C_{0} G^{\star}\right) \leq \sum_{(k, i) \in E(\Gamma)}\left\|Z^{\star \star} e_{k i}-O_{i}^{\star \star} \bar{x}_{k}\right\|^{2} .
$$

To bring in the error term, we write

$$
Z^{\star \star} e_{k i}-O_{i}^{\star \star} \bar{x}_{k}=Z^{\star \star} e_{k i}-O_{i}^{\star \star} x_{k, i}+O_{i}^{\star \star} \epsilon_{k, i},
$$


and use $\|x+y\|^{2} \leq 2\left(\|x\|^{2}+\|y\|^{2}\right)$ to get

$$
\operatorname{Tr}\left(C_{0} G^{\star}\right) \leq 2 \sum_{(k, i) \in E}\left\|Z^{\star \star} e_{k i}-O_{i}^{\star \star} x_{k, i}\right\|^{2}+2|E(\Gamma)| \varepsilon^{2} .
$$

Finally, using the optimality of $\left(Z^{\star \star}, O^{\star \star}\right)$ for $(47)$, we have

$$
\sum_{(k, i) \in E(\Gamma)}\left\|Z^{\star \star} e_{k i}-O_{i}^{\star \star} x_{k, i}\right\|^{2} \leq \sum_{(k, i) \in E(\Gamma)}\left\|Z_{0} e_{k i}-I_{d} x_{k, i}\right\|^{2} \leq|E(\Gamma)| \varepsilon^{2} .
$$

The desired result follows from (48), 49), and (50).

Finally, we note that $\operatorname{Tr}(T)$ can be used to bound the difference between the Gram matrices.

Proposition 20 (Trace bound). $\left\|W^{\star T} W^{\star}-G_{0}\right\|_{F} \leq 2 \sqrt{2 M d \operatorname{Tr}(T)}$.

Proof. We will heavily use decomposition (45) and its properties. Let $G^{\star}=G_{0}+\Delta$. By triangle inequality,

$$
\begin{aligned}
\left\|W^{\star T} W^{\star}-G_{0}\right\|_{F} & \leq\left\|\sum_{i=d+1}^{M d} \lambda_{i}\left(G^{\star}\right) u_{i} u_{i}^{T}\right\|_{F}+\|\Delta\|_{F} \\
& =\left\|\operatorname{diag}\left(\lambda_{d+1}\left(G^{\star}\right), \ldots, \lambda_{M d}\left(G^{\star}\right)\right)\right\|_{F}+\|\Delta\|_{F} .
\end{aligned}
$$

Moreover, since the bottom eigenvalues of $G_{0}$ are zero, it follows from Lemma 17 that the norm of the diagonal matrix is bounded by $\|\Delta\|_{F}$. Therefore,

$$
\left\|W^{\star T} W^{\star}-G_{0}\right\|_{F} \leq 2\|\Delta\|_{F} .
$$

Fix $\left\{s_{d+1}, \ldots, s_{M d}\right\}$ to be some orthonormal basis of $\bar{S}$. For arbitrary $A \in \mathbb{R}^{M d}$, let

$$
A(p, q)=s_{p}^{T} A s_{q} \quad(1 \leq p, q \leq M d) .
$$

That is, $(A(p, q))$ are the coordinates of $A$ in the basis $\left\{s_{1}, \ldots, s_{d}\right\} \cup\left\{s_{d+1}, \ldots, s_{M d}\right\}$. Decompose $\Delta=P+Q+T$ as in (45). Note that $P, Q$, and $T$ are represented in the above basis as follows: $P$ is supported on the upper $d \times d$ diagonal block, $T$ is supported on the lower $(M-1) d \times(M-1) d$ diagonal block, and $Q$ on the off-diagonal blocks. The matrix $G_{0}$ is diagonal in this representation.

We can bound $\|P\|_{F}$ using Lemma 18 .

$$
\|P\|_{F}^{2}=M^{2}\left\|P_{11}\right\|_{F}^{2}=\left\|\sum_{l=1}^{M} T_{l l}\right\|_{F}^{2} \leq\left[\operatorname{Tr}\left(\sum_{l=1}^{M} T_{l l}\right)\right]^{2}=\operatorname{Tr}(T)^{2},
$$

where we have used the properties $T \succeq 0$ and $T_{l l} \succeq 0(1 \leq l \leq M)$. In particular,

$$
\|T\|_{F} \leq \operatorname{Tr}(T) .
$$

On the other hand, since $G_{0}+\Delta \succeq 0$, we have $\left(G_{0}+\Delta\right)(p, q)^{2} \leq\left(G_{0}+\Delta\right)(p, p)\left(G_{0}+\right.$ $\Delta)(q, q)$. Therefore,

$$
\|Q\|_{F}^{2}=2 \sum_{p=1}^{d} \sum_{q=d+1}^{M d} Q(p, q)^{2} \leq 2 \sum_{p=1}^{d}\left(G_{0}+\Delta\right)(p, p) \sum_{q=d+1}^{M d} T(q, q) .
$$

Notice that $0=\operatorname{Tr}(\Delta)=\operatorname{Tr}(T)+\operatorname{Tr}(P)$. Therefore,

$$
\|Q\|_{F}^{2} \leq 2 M d \operatorname{Tr}(T)-2 \operatorname{Tr}(T)^{2} .
$$

Combining (51), 52), (54), and (53), we get the desired bound. 
Putting together (40) with Propositions (14), (19), and (20), we arrive at Theorem (13).

\section{NUMERICAL EXPERIMENTS}

We now present some numerical results on multipatch registration using GRET-SPEC and GRET-SDP. In particular, we study the exact recovery and stability properties of the algorithm. We define the reconstruction error in terms of the root-mean-square deviation (RMSD) given by

$$
\operatorname{RMSD}=\min _{\Omega \in \mathbb{O}(d), t \in \mathbb{R}^{d}}\left[\frac{1}{N} \sum_{k=1}^{N}\left\|Z_{k}^{\star}-\Omega \bar{x}_{k}-t\right\|^{2}\right]^{1 / 2} .
$$

In other words, the RMSD is calculated after registering (aligning) the original and the reconstructed configurations. We use the SVD-based algorithm [2] for this purpose.

Experiment 1. We first consider a few examples concerning the registration of three patches in $\mathbb{R}^{2}$, where we vary $\Gamma$ by controlling the number of points in the intersection of the patches. We work with the clean data in 25) and demonstrate exact recovery (up to numerical precision) for different $\Gamma$.

In the left plot in Figure 4 , we consider a patch system with $N=10$ points. The points that belong to two or more patches are marked red, while the rest are marked black. The patches taken in the order $P_{1}, P_{2}, P_{3}$ form a lateration in this case. As predicted by Corollary 6 and Theorem 7. the rank of the patch-stress matrix $C_{0}$ for this system must be $2(3-1)=4$. This is indeed confirmed by our experiment. We expect GRET-SPEC and GRET-SDP to recover the exact configuration. Indeed, we get a very small RMSD of the order of 1e-7 in this case. As shown in the figure, the reconstructed coordinates obtained using GRET-SDP perfectly match the original ones after alignment.

We next consider the example shown in the center plot in Figure 4 The patch system is not laterated in this case, but the rank of $C_{0}$ is 4 . Again we obtain a very small RMSD of the order 1e-7 for this example. This example demonstrates that lateration is not necessary for exact recovery.
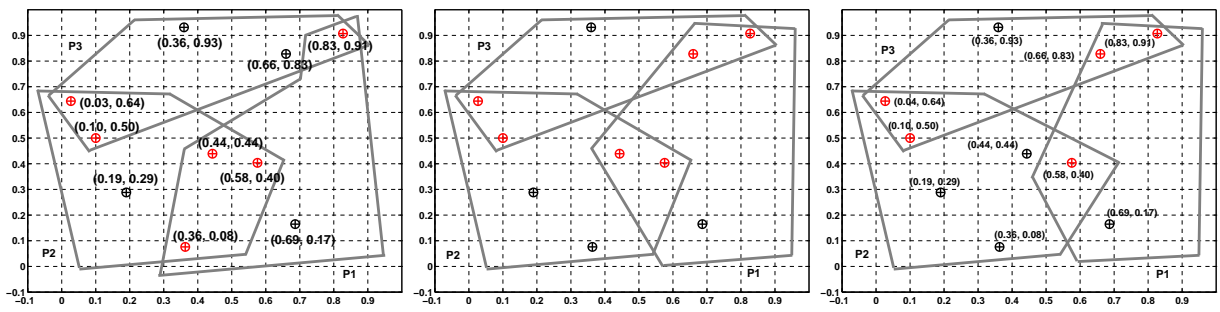

FIgURE 4. Instances of a three-patch systems in $\mathbb{R}^{2}$. Left: Patch system is laterated. Center: Patch system is not laterated but for which $C_{0}$ has rank 4 . Right: The body graph is universally rigid but $\operatorname{rank}\left(C_{0}\right)=3$. The original coordinates are marked with $\circ$, and the coordinates reconstructed by GRET-SDP with + . 
In the next example, we show that the condition $\operatorname{rank}\left(C_{0}\right)=(M-1) d$ is not necessary for exact recovery using GRET-SDP. To do so, we use the fact that the universal rigidity of the body graph is both necessary and sufficient for exact recovery. Consider the example shown in the right plot in Figure 4 . This has barely enough points in the patch intersections to make the body graph universally rigid. Experiments confirm that we have exact recovery in this case. However, it can be shown that $\operatorname{rank}\left(C_{0}\right)<(M-1) d=4$.

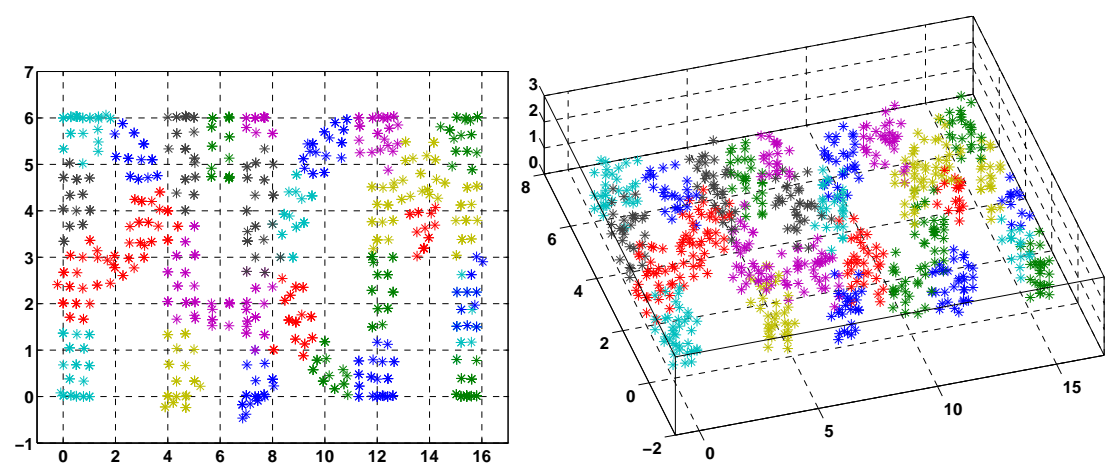

FIGURE 5. Disjoint clusters for the PACM point cloud. Each cluster is marked with a different color. The clusters are augmented to form overlapping patches which are then registered using GRET-SDP.

Experiment 2. We now consider the structured PACM data in $\mathbb{R}^{3}$ shown in Figure 5 The are a total of 799 points in this example that are obtained by sampling the 3-dimensional PACM logo [17, 20]. To begin with, we divide the point cloud into $M=30$ disjoint pieces (clusters) as shown in the figure. We augment each cluster into a patch by adding points from neighboring clusters. We ensure that there are sufficient common points in the patch system so that $C_{0}$ has rank $(M-1) d=87$. We generate the measurements using the bounded noise model in (34). In particular, we perturb the clean coordinates using uniform noise over the hypercube $[-\varepsilon, \varepsilon]^{d}$. For the noiseless setting, the RMSD's obtained using GRET-SPEC and GRET-SDP are $3.3 \mathrm{e}-11$ and $1 \mathrm{e}-6$. The respective RMSD's when $\varepsilon=0.5$ are 1.4743 and 0.3823 . The results are shown in Figure 6.

Experiment 3. In the final experiment, we demonstrate the stability of GRET-SDP and GRET-SPEC by plotting the RMSD against the noise level for the PACM data. We use the noise model in (34) and vary $\varepsilon$ from 0 to 2 in steps of 0.1 . For a fixed noise level, we average the RMSD over 20 noise realizations. The results are reported in the bottom plot in Figure 7. We see that the RMSD increases gracefully with the noise level. The result also shows that the semidefinite relaxation is more stable than spectral relaxation, particularly at large noise levels. Also shown in the figure are the RMSD obtained using GRET-MANOPT with the solutions of GRET-SPEC and GRET-SDP as initialization. In particular, we used the trust region method provided in the Manopt toolbox [10] for solving the manifold optimization $\left(\mathrm{P}_{0}\right)$. For either initialization, we notice some improvement from the plots. It is clear that the manifold method relies heavily on the initialization, which is not surprising. 

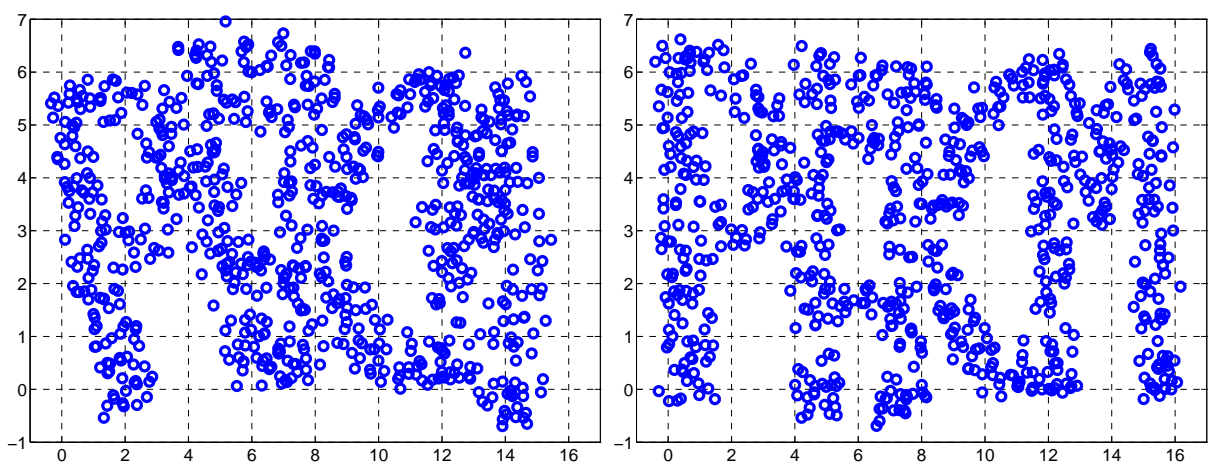

FIGURE 6. Reconstruction of the PACM data from corrupted patch coordinates $(\varepsilon=0.5)$. Left: GRET-SPEC, RMSD $=1.4743$. Right: GRET-SDP, RMSD $=0.3823$. The measurements were generated using the noise model in (34).
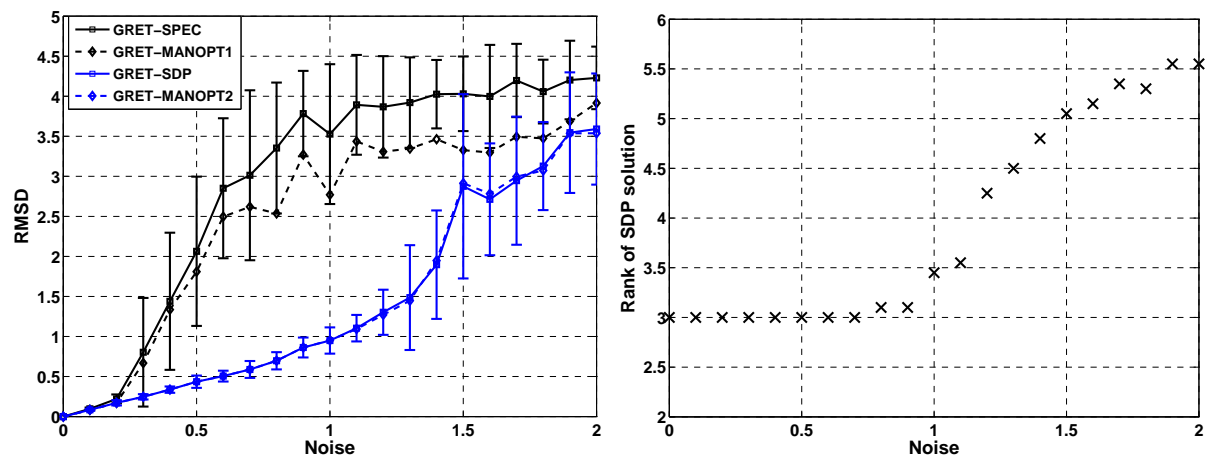

FIGURE 7. Left: RMSD versus noise level $\varepsilon$. GRET-MANOPT1 (resp. GRET-MANOPT2) is the result obtained by refining the output of GRET-SPEC (resp. GRET-SDP) using manifold optimization.

Right: Rank of $G^{\star}$ in GRET-SDP.

Finally, we plot the rank of the SDP solution $G^{\star}$ and notice an interesting phenomenon. Up to a certain noise level, $G^{\star}$ has the desired rank and rounding is not required. This means that the relaxation gap is zero for the semidefinite relaxation, and that we can solve the original non-convex problem using GRET-SDP up to a certain noise threshold. It is therefore not surprising that the RMSD shows no improvement after we refine the SDP solution using manifold optimization. We have noticed that the rank of the SDP solution is stable with respect to noise for other numerical experiments as well (not reported here).

\section{DisCUSSION}

There are several directions along which the present work could be extended and refined. We summarize some of these below. 
(1) Rank Recovery. Exhaustive numerical simulations (see, for example, Figure 7) show us that the proposed program is quite stable as far as rank recovery is concerned. By rank recovery, we mean that $\operatorname{rank}\left(G^{\star}\right)=d$. In this case, the relaxation gap is zero - we have actually solved the original non-convex problem. We have performed numerical experiments in which we fix some $\Gamma$ for which $\operatorname{rank}(C)=(M-1) d$, and gradually increase the noise in the measurements as per the model in (34). When the noise is zero, we recover the exact Gram matrix that has rank $d$. What is interesting is that the program keeps returning a rank- $d$ solution up to a certain noise level. In other words, we observe a phase transition phenomenon in which $\operatorname{rank}\left(G^{\star}\right)$ is consistently $d$ up to a certain noise threshold. This threshold seems to depend on the number of points in the intersection of the patches, which is perhaps not surprising. A precise understanding of this phase transition in terms of the properties of $\Gamma$ would be an interesting study.

(2) Conditions on $\Gamma$. We have seen that the universal rigidity of the body graph (derived from $\Gamma$ ) is both necessary and sufficient for exact recovery using GRET-SDP. However, to test unique rigidity, we need to run a semidefinite program [56]. Unfortunately, the complexity of this program is much more than GRET-SDP itself. This led us to consider the rank criteria that could be tested efficiently. The rank test is nonetheless not necessary for exact recovery, and weaker conditions can be found. In particular, an interesting question is whether we could find an efficiently-testable condition that would hold true for the extreme example in Figure 4 , in which $\Gamma$ fails the rank test?

(3) Tighter Bounds. The stability in Theorem 13 was for the bounded noise model, which made the subsequent analysis quite straightforward. The goal was to establish that the reconstruction error is within $C \varepsilon$ for some constant $C$ independent of the noise. In particular, the bounds in Theorem 13 are quite loose. One possible direction would be to consider a stochastic noise model with statistically independent perturbations to tighten the bound.

(4) Anchor Points. In sensor network localization, one has to infer the coordinates of sensors from the knowledge of distances between sensors and its geometric neighbors. In distributed approaches to sensor localization [16, 9], one is faced exactly with the multipatch registration problem described in this paper. Besides the distance information, one often has the added knowledge of the precise positions of selected sensors known as anchors [8]. This is often by design and is used to improve the localization accuracy. The question is can we incorporate the anchor constraints into the present registration algorithm? One possible way of leveraging the existing framework is to introduce an additional patch (called anchor patch) for the anchor points. The anchor coordinates are assigned to the points in the anchor patch (treating them as local coordinates). This gives us an augmented bipartite graph $\Gamma_{a}$ which has one more patch vertex than $\Gamma$, and extra edges connecting the anchor patch to the anchor vertices. We then proceed exactly as before, that is, we solve for the global coordinates of both the anchor and non-anchor points given the measurements on $\Gamma_{a}$. 


\section{ACKNOWLEDGEMENTS}

K. N. Chaudhury was partially supported by the Swiss National Science Foundation under Grant PBELP2-135867 and by Award Number R01GM090200 from the National Institute of General Medical Sciences while he was at Princeton University, and more recently by a Startup Grant from the Indian Institute of Science.

Y. Khoo was partially supported by Award Number R01 GM090200-01 from the National Institute for Health.

A. Singer was partially supported by Award Numbers FA9550-13-1-0076 and FA9550-12-1-0317 from AFOSR, Award Number R01GM090200 from the National Institute of General Medical Sciences, and Award Number LTR DTD 06-05-2012 from the Simons Foundation.

The authors are grateful to the anonymous referees for their comments and suggestions. They particularly thank the referees for pointing out references [68, 25] and their relation to the present work. The authors also thank Mihai Cucuringu, Lanhui Wang, and Afonso Bandeira for useful discussions, and Nicolas Boumal for advice on the usage of the Manopt toolbox.

\section{REFERENCES}

[1] P.-A. Absil, R. Mahony, And R. Sepulchre, Optimization Algorithms on Matrix Manifolds, Princeton University Press, 2009.

[2] K. S. Arun, T. S. HuAnG, AND S. D. Blostein, Least-squares fitting of two $3 d$ point sets, IEEE Transactions on Pattern Analysis and Machine Intelligence, (1987), pp. 698-700.

[3] A. S. BAndeIRA, C. KenNeDY, AND A. Singer, Approximating the little Grothendieck problem over the orthogonal group, arXiv:1308.5207, (2013).

[4] A. S. BANDeIRA, A. Singer, AND D. A. SPIElman, A Cheeger inequality for the graph connection Laplacian, SIAM Journal on Matrix Analysis and Applications, 34 (2013), pp. 1611-1630.

[5] S. R. BECKER, E. J. CANDÈS, AND M. C. GRANT, Templates for convex cone problems with applications to sparse signal recovery, Mathematical Programming Computation, 3 (2011), pp. 165-218.

[6] P. J. BESL AND N. D. MCKAY, A method for registration of $3 d$ shapes, IEEE Transactions on Pattern Analysis and Machine Intelligence, 14 (1992), pp. 239-256.

[7] R. Bhatia, Matrix Analysis, vol. 169, Springer, 1997.

[8] P. BISWAS, T.-C. LIANG, K.-C. TOH, Y. YE, AND T.-C. WANG, Semidefinite programming approaches for sensor network localization with noisy distance measurements, IEEE Transactions on Automation Science and Engineering, 3 (2006), pp. 360-371.

[9] P. BISWAS, K.-C. TOH, AND Y. YE, A distributed SDP approach for large-scale noisy anchor-free graph realization with applications to molecular conformation, SIAM Journal on Scientific Computing, 30 (2008), pp. 1251-1277.

[10] N. Boumal, B. Mishra, P.-A. Absil, And R. Sepulchre, Manopt: A Matlab toolbox for optimization on manifolds, The Journal of Machine Learning Research, (2014). Accepted for publication.

[11] S. BURER AND R. D. C. MONTEIRO, A nonlinear programming algorithm for solving semidefinite programs via low-rank factorization, Mathematical Programming, 95 (2003), pp. 329-357.

[12] E. CANDES AND B. RECHT, Exact matrix completion via convex optimization, Foundations of Computational Mathematics, 9 (2009), pp. 717-772.

[13] E. J. CANDES, T. STROHMER, AND V. VORONINSKI, Phaselift: Exact and stable signal recovery from magnitude measurements via convex programming, Communications on Pure and Applied Mathematics, 66 (2013), pp. 1241-1274.

[14] F. R. K. CHUNG, Spectral Graph Theory, vol. 92, American Mathematical Society, 1997.

[15] R. Connelly, Rigidity and energy, Inventiones Mathematicae, 66 (1982), pp. 11-33.

[16] M. CUCURINGU, Y. LIPMAN, AND A. SINGER, Sensor network localization by eigenvector synchronization over the euclidean group, ACM Transactions on Sensor Networks, 8 (2012), p. 19.

[17] M. CUCURINGU, A. SINGER, AND D. COWBURN, Eigenvector synchronization, graph rigidity and the molecule problem, Information and Inference, 1 (2012), pp. 21-67. 
[18] A. Edelman, T. A. ARIAS, AND S. T. SMith, The geometry of algorithms with orthogonality constraints, SIAM Journal on Matrix Analysis and Applications, 20 (1998), pp. 303-353.

[19] K. FAn AND A. J. Hoffman, Some metric inequalities in the space of matrices, Proceedings of the American Mathematical Society, 6 (1955), pp. 111-116.

[20] X. FANG AND K.-C. TOH, Using a distributed SDP approach to solve simulated protein molecular conformation problems, in Distance Geometry, Springer, 2013, pp. 351-376.

[21] O. D. FAUGERAS AND M. HEBERT, The representation, recognition, and locating of $3 d$ objects, International Journal of Robotics Research, 5 (1986), pp. 27-52.

[22] D. Garber And E. HazAN, Approximating semidefinite programs in sublinear time, in Advances in Neural Information Processing Systems, 2011, pp. 1080-1088.

[23] M. X. GoEMANS AND D. P. WiLliAMSON, Improved approximation algorithms for maximum cut and satisfiability problems using semidefinite programming, Journal of the ACM, 42 (1995), pp. 1115-1145.

[24] G. H. Golub AND C. F. V. LOAN, Matrix Computations, Johns Hopkins University Press, 1996.

[25] S. GorTLer, C. Gotsman, L. LiU, AND D. Thurston, On affine rigidity, Journal of Computational Geometry, 4 (2013), pp. 160-181.

[26] S. J. Gortler, A. D. HeAly, And D. P. Thurston, Characterizing generic global rigidity, American Journal of Mathematics, 132 (2010), pp. 897-939.

[27] S. J. GORTLER AND D. P. THURSTON, Characterizing the universal rigidity of generic frameworks, arXiv preprint arXiv:1001.0172, (2009).

[28] J. C. Gower And G. B. DijKsterhuis, Procrustes Problems, vol. 3, Oxford University Press Oxford, 2004.

[29] M. GRANT, S. BOYD, AND Y. YE, CVX: Matlab software for disciplined convex programming, 2008.

[30] C. HelmberG, F. Rendl, R. J. VAnderbei, AND H. Wolkowicz, An interior-point method for semidefinite programming, SIAM Journal on Optimization, 6 (1996), pp. 342-361.

[31] B. HENDRICKSON, Conditions for unique graph realizations, SIAM Journal on Computing, 21 (1992), pp. 65-84.

[32] J. N. HIGHAM, Computing the polar decomposition - with applications, SIAM Journal on Scientific and Statistical Computing, 7 (1986), pp. 1160-1174.

[33] N.-D. Ho AND P. V. DOOREN, On the pseudo-inverse of the Laplacian of a bipartite graph, Applied Mathematics Letters, 18 (2005), pp. 917-922.

[34] B. K. P. HORN, Closed-form solution of absolute orientation using unit quaternions, JOSA A, 4 (1987), pp. 629-642.

[35] S. D. HOWARD, D. COCHRAN, W. MORAN, AND F. R. COHEN, Estimation and registration on graphs, arXiv:1010.2983, (2010).

[36] Q.-X. HuANG AND L. GUIBAS, Consistent shape maps via semidefinite programming, in Eurographics Symposium on Geometry Processing, vol. 32, 2013, pp. 177-186.

[37] A. JAVANMARD AND A. MONTANARI, Localization from incomplete noisy distance measurements, Foundations of Computational Mathematics, 13 (2013), pp. 297-345.

[38] M. JOURNEE, F. BACH, P.-A. ABSIL, AND R. SEPULCHRE, Low-rank optimization on the cone of positive semidefinite matrices, SIAM Journal on Optimization, 20 (2010), pp. 2327-2351.

[39] J. B. KELLER, Closest unitary, orthogonal and hermitian operators to a given operator, Mathematics Magazine, 48 (1975), pp. 192-197.

[40] S. KRishnan, P. Y. LEE, J. B. MOORE, AND S. VenKatAsubramanian, Global registration of multiple $3 d$ point sets via optimization-on-a-manifold, Eurographics Symposium on Geometry Processing, (2005), pp. 187-197.

[41] G. Lerman, M. MCCOY, J. A. TROPP, AND T. ZHANG, Robust computation of linear models, or how to find a needle in a haystack, arXiv:1202.4044, (2012).

[42] R.-C. LI, New perturbation bounds for the unitary polar factor, SIAM Journal on Matrix Analysis and Applications, 16 (1995), pp. 327-332.

[43] L. LOVÁsZ AND A. SCHRIJVER, Cones of matrices and set-functions and 0-1 optimization, SIAM Journal on Optimization, 1 (1991), pp. 166-190.

[44] L. MIRSKY, Symmetric gauge functions and unitarily invariant norms, The Quarterly Journal of Mathematics, 11 (1960), pp. 50-59.

[45] N. J. Mitra, N. Gelfand, H. Pottmann, And L. Guibas, Registration of point cloud data from a geometric optimization perspective, in Eurographics Symposium on Geometry Processing, 2004, pp. 22-31.

[46] A. NAOR, O. REGEV, AND T. VIDICK, Efficient rounding for the noncommutative rothendieck inequality, in Proceedings of the 45th Annual ACM Symposium on Theory of Computing, 2013, pp. 71-80. 
[47] A. NemiRovSKI, Sums of random symmetric matrices and quadratic optimization under orthogonality constraints, Mathematical Programming, 109 (2007), pp. 283-317.

[48] Y. NESTEROV, Semidefinite relaxation and nonconvex quadratic optimization, Optimization methods and software, 9 (1998), pp. 141-160.

[49] H. POtTMANN, Q.-X. HUANG, Y.-L. YANG, AND S.-M. HU, Geometry and convergence analysis of algorithms for registration of $3 d$ shapes, International Journal of Computer Vision, 67 (2006), pp. 277296.

[50] G. RANJAN, Z.-L. ZHANG, AND D. BOLEY, Incremental computation of pseudo-inverse of Laplacian: Theory and applications, arXiv:1304.2300, (2013).

[51] S. RUSINKIEWICZ AND M. LEVOY, Efficient variants of the ICP algorithm, in Proceedings of the Third International Conference on 3d Digital Imaging and Modeling, 2001, pp. 145-152.

[52] G. C. SHARP, S. W. LEE, AND D. K. WEHE, Multiview registration of $3 d$ scenes by minimizing error between coordinate frames, in Proceedings of the 7th European Conference on Computer Vision-Part II, Springer-Verlag, 2002, pp. 587-597.

[53] A. SINGER, Angular synchronization by eigenvectors and semidefinite programming, Applied and Computational Harmonic Analysis, 30 (2011), pp. 20-36.

[54] A. SINGER AND M. CUCURINGU, Uniqueness of low-rank matrix completion by rigidity theory, SIAM Journal on Matrix Analysis and Applications, 31(4) (2010), pp. 1621-1641.

[55] A. M.-C. So, Moment inequalities for sums of random matrices and their applications in optimization, Mathematical Programming, 130 (2011), pp. 125-151.

[56] A. M.-C. SO AND Y. YE, Theory of semidefinite programming for sensor network localization, Mathematical Programming, 109 (2007), pp. 367-384.

[57] D. A. SPIElmAN AND S.-H. TENG, Nearly-linear time algorithms for graph partitioning, graph sparsification, and solving linear systems, in Proceedings of the Thirty-sixth Annual ACM Symposium on Theory of Computing, 2004, pp. 81-90.

[58] K.-C. TOH, M. J. TODD, AND R. H. TUTUNCU, SDPT3 - A Matlab software package for semidefinite programming, version 1.3, Optimization Methods and Software, 11 (1999), pp. 545-581.

[59] T. TZENEVA, Global alignment of multiple $3 d$ scans using eigevector synchronization, Senior Thesis, Princeton University (supervised by S. Rusinkiewicz and A. Singer), (2011).

[60] L. VANDENBERGHE AND S. BOYD, Semidefinite programming, SIAM review, 38 (1996), pp. 49-95.

[61] N. K. VisHNOI, $L x=b$, Foundations and Trends in Theoretical Computer Science, 8 (2012), pp. 1-141.

[62] I. WALDSPURGer, A. D'Aspremont, AND S. MALLAT, Phase recovery, maxcut and complex semidefinite programming, Mathematical Programming, (2013), pp. 1-35.

[63] L. WANG AND A. SINGER, Exact and stable recovery of rotations for robust synchronization, Information and Inference: A Journal of the IMA, 2 (2013), pp. 145-193.

[64] Z. Wen, D. GOlDFARB, S. MA, AND K. SCHEINBERG, Block coordinate descent methods for semidefinite programming, in Handbook on Semidefinite, Conic and Polynomial Optimization, Springer, 2012, pp. $533-564$.

[65] J. A. Williams And M. Bennamoun, Simultaneous registration of multiple point sets using orthonormal matrices, in Proceedings of IEEE International Conference on Acoustics, Speech, and Signal Processing, vol. 6, 2000, pp. 2199-2202.

[66] H. WOLKOWICZ AND M. F. ANJOS, Semidefinite programming for discrete optimization and matrix completion problems, Discrete Applied Mathematics, 123 (2002), pp. 513-577.

[67] H. Wolkowicz, R. SAigal, And L. VAndenberghe, Handbook of Semidefinite Programming: Theory, Algorithms, and Applications, vol. 27, Kluwer Academic Pub, 2000.

[68] H. ZHA AND Z. ZHANG, Spectral properties of the alignment matrices in manifold learning, SIAM review, 51 (2009), pp. 545-566.

[69] L. Zhang, L. LiU, C. Gotsman, And S. Gortler, An As-Rigid-As-Possible approach to sensor network localization, ACM Transactions on Sensor Networks, 6 (2010), p. 35.

[70] L. ZHI-QuAN, M. WING-KIN, A.-C. SO, Y. YE, AND S. ZHANG, Semidefinite relaxation of quadratic optimization problems, IEEE Signal Processing Magazine, 27 (2010), pp. 20-34.

[71] Z. ZHU, A. M.-C. SO, AND Y. YE, Universal rigidity and edge sparsification for sensor network localization, SIAM Journal on Optimization, 20 (2010), pp. 3059-3081. 


\section{TECHNICAL PROOFS}

In this Section, we give the proof of Propositions 11 and 14 and Lemmas 15 and 16.

8.1. Proof of Proposition 11. We are done if we can show that there exists a bijection between the nullspace of $C$ and that of $C_{0}$. To do so, we note that the associated quadratic forms can be expressed as

$$
u^{T} C u=\min _{z \in \mathbb{R}^{1 \times N+M}} \sum_{(k, i) \in E(\Gamma)}\left\|z e_{k i}-u_{i}^{T} x_{k, i}\right\|^{2},
$$

and

$$
v^{T} C_{0} v=\min _{z \in \mathbb{R}^{1 \times N+M}} \sum_{(k, i) \in E(\Gamma)}\left\|z e_{k i}-v_{i}^{T} \bar{x}_{k}\right\|^{2} .
$$

Here $u_{1}, \ldots, u_{M}$ are the $d \times 1$ blocks of the vector $u \in \mathbb{R}^{M d \times 1}$.

Now, it follows from 25 that there is a one-to-one correspondence between $u$ and $v$, namely

$$
u_{i}=\bar{O}_{i} v_{i} \quad(1 \leq i \leq M)
$$

such that $u^{T} C u=v^{T} C_{0} v$. In other words, the null space of $C$ is related to the null space of $C_{0}$ through an orthogonal transform, as was required to be shown.

8.2. Proof of Proposition 14. Without loss of generality, we assume that the smallest Euclidean ball that encloses the clean configuration $\left\{\bar{x}_{1}, \ldots, \bar{x}_{N}\right\}$ is centered at the origin, that is,

$$
\left\|\bar{x}_{k}\right\| \leq R \quad(1 \leq k \leq N) .
$$

Let $B_{0}$ be the matrix $B$ in (12) computed from the clean measurements, i.e., from (34) with $\varepsilon=0$. Let $B_{0}+H$ be the same matrix obtained from (34) for some $\varepsilon>0$. Recall that $Z_{0}=O_{0} B_{0} L^{\dagger}$ (by the centering assumption in (35)). Therefore, $\left\|Z^{\star}-\Theta Z_{0}\right\|_{F}=\left\|O^{\star}\left(B_{0}+H\right) L^{\dagger}-\Theta O_{0} B_{0} L^{\dagger}\right\|_{F}=\left\|\left(O^{\star}-\Theta O_{0}\right) B_{0} L^{\dagger}+O^{\star} H L^{\dagger}\right\|_{F}$.

By triangle inequality,

$$
\left\|Z^{\star}-\Theta Z_{0}\right\|_{F} \leq\left\|O^{\star}-\Theta O_{0}\right\|_{F}\left\|B_{0} L^{\dagger}\right\|_{F}+\left\|O^{\star} H L^{\dagger}\right\|_{F},
$$

Now

$$
\left\|B_{0} L^{\dagger}\right\|_{F} \leq\left\|L^{\dagger}\right\|_{\mathrm{sp}}\left\|B_{0}\right\|_{F}=\frac{1}{\lambda_{2}(L)}\left\|B_{0}\right\|_{F}
$$

where $\lambda_{2}(L)$ is the smallest non-zero eigenvalue of $L$. On the other hand,

$$
B_{0}=\sum_{(k, i) \in E(\Gamma)}\left(e_{i}^{M} \otimes I_{d}\right) \bar{x}_{k} e_{k i}^{T} .
$$

Using Cauchy-Schwarz and (56), we get

$$
\begin{aligned}
\left\|B_{0}\right\|_{F}^{2} & =\sum_{(k, i) \in E(\Gamma)} \sum_{(l, j) \in E(\Gamma)} \operatorname{Tr}\left(e_{k i} \bar{x}_{k}^{T}\left(e_{i}^{M} \otimes I_{d}\right)^{T}\left(e_{j}^{M} \otimes I_{d}\right) \bar{x}_{l} e_{l j}^{T}\right) \\
& =\sum_{(k, i) \in E(\Gamma)} \sum_{(l, i) \in E(\Gamma)} \bar{x}_{k}^{T} \bar{x}_{l} e_{k i}^{T} e_{l i} . \\
& \leq \sum_{(k, i) \in E(\Gamma)} 2 R^{2}+\sum_{(k, i) \in E(\Gamma)} \sum_{(l, i) \in E(\Gamma)} R^{2} .
\end{aligned}
$$


Therefore,

$$
\left\|B_{0} L^{\dagger}\right\|_{F} \leq \lambda_{2}(L)^{-1} \sqrt{2+N}|E(\Gamma)|^{1 / 2} R .
$$

As for the other term in (57), we can write

$$
\left\|O^{\star} H L^{\dagger}\right\|_{F} \leq\left\|L^{\dagger}\right\|_{\mathrm{sp}}\left\|O^{\star} H\right\|_{F}=\lambda_{2}(L)^{-1}\left\|O^{\star} H\right\|_{F} .
$$

Now

$$
O^{\star} H=O^{\star}\left(B-B_{0}\right)=\sum_{(k, i) \in E(\Gamma)} O_{i}^{\star} \epsilon_{k, i} e_{k i}^{T} .
$$

Therefore, using Cauchy-Schwarz, the orthonormality of the columns of $O_{i}^{\star \prime}$ s, and the noise model (34), we get

$$
\begin{aligned}
\left\|O^{\star} H\right\|_{F}^{2} & =\sum_{(k, i) \in E(\Gamma)} \sum_{(l, j) \in E(\Gamma)}\left(O_{i}^{\star} \epsilon_{k, i}\right)^{T}\left(O_{j}^{\star} \epsilon_{l, j}\right) e_{k i}^{T} e_{l i} \\
& \leq \sum_{(k, i) \in E(\Gamma)} 2 \varepsilon^{2}+\sum_{(k, i) \in E(\Gamma)} \sum_{(l, i) \in E(\Gamma)} \varepsilon^{2}+\sum_{(k, i) \in E(\Gamma)} \sum_{(k, j) \in E(\Gamma)} \varepsilon^{2} .
\end{aligned}
$$

This gives us

$$
\left\|O^{\star} H L^{\dagger}\right\|_{F} \leq \sqrt{2+N+M}|E(\Gamma)|^{1 / 2} \lambda_{2}(L)^{-1} \varepsilon .
$$

Combining (57), (58), and (59), we get the desired estimate.

8.3. Proof of Lemma 15. The proof is mainly based on the observation that if $u$ and $v$ are unit vectors and $0 \leq u^{T} v \leq 1$, then

$$
\|u-v\| \leq\left\|u u^{T}-v v^{T}\right\|_{F} .
$$

Indeed,

$$
\left\|u u^{T}-v v^{T}\right\|_{F}^{2}=\operatorname{Tr}\left(u u^{T}+v v^{T}-2\left(u^{T} v\right)^{2}\right) \geq \operatorname{Tr}\left(u u^{T}+v v^{T}-2 u^{T} v\right)=\|u-v\|^{2} .
$$

To use this result in the present setting, we use the theory of principal angles [7, Ch. 7.1]. This tells us that, for the orthonormal systems $\left\{u_{1}, \ldots, u_{d}\right\}$ and $\left\{s_{1}, \ldots, s_{d}\right\}$, we can find $\Omega_{1}, \Omega_{2} \in \mathbb{O}(M d)$ such that

(1) $\Omega_{1}\left[u_{1} \cdots u_{d}\right]=\left[u_{1} \cdots u_{d}\right] \Theta_{1}^{T}$ where $\Theta_{1} \in \mathbb{O}(d)$,

(2) $\Omega_{2}\left[s_{1} \cdots s_{d}\right]=\left[s_{1} \cdots s_{d}\right] \Theta_{2}^{T}$ where $\Theta_{2} \in \mathbb{O}(d)$,

(3) $\left(\Omega_{1} s_{i}\right)^{T}\left(\Omega_{2} u_{j}\right)=0$ for $i \neq j$, and $0 \leq\left(\Omega_{1} s_{i}\right)^{T}\left(\Omega_{2} u_{i}\right) \leq 1$ for $1 \leq i \leq d$.

Here $\Theta_{1}$ and $\Theta_{2}$ are the orthogonal transforms that map $\left\{u_{1}, \ldots, u_{d}\right\}$ and $\left\{s_{1}, \ldots, s_{d}\right\}$ into the corresponding principal vectors.

Using properties 1 and 2 and the fact ${ }^{4}$ that $\alpha_{i} \leq M$, we can write

$$
\sqrt{M}\left\|\Theta_{1} W^{\star}-\Theta_{2} O_{0}\right\|_{F} \leq\left\|\Omega_{1}\left[\alpha_{1} u_{1} \cdots \alpha_{d} u_{d}\right]-M \Omega_{2}\left[s_{1} \cdots s_{d}\right]\right\|_{F}+\left[\sum_{i=1}^{d}\left(M-\alpha_{i}\right)^{2}\right]^{1 / 2} \text {. }
$$

\footnotetext{
${ }^{4}$ To see why the eigenvalues of $G^{\star}$ are at most $M$ (the authors thank Afonso Bandeira for suggesting this), note that by the SDP constraints, for every block $G_{i j}$,

$$
u^{T} G_{i j} v \leq\left(\|u\|^{2}+\|v\|^{2}\right) / 2 \quad\left(u, v \in \mathbb{R}^{d}\right) .
$$

Let $x=\left(x_{1}, \ldots, x_{M}\right)$ where each $x_{i} \in \mathbb{R}^{d}$. Then

$$
x^{T} G x=\sum_{i, j} x_{i}^{T} G_{i j} x_{j} \leq \sum_{i, j}\left(\left\|x_{i}\right\|^{2}+\left\|x_{j}\right\|^{2}\right) / 2=M\|x\|^{2} .
$$
}


Moreover, by triangle inequality,

$\left\|\Omega_{1}\left[\alpha_{1} u_{1} \cdots \alpha_{d} u_{d}\right]-M \Omega_{2}\left[s_{1} \cdots s_{d}\right]\right\|_{F} \leq M\left\|\Omega_{1}\left[u_{1} \cdots u_{d}\right]-\Omega_{2}\left[s_{1} \cdots s_{d}\right]\right\|_{F}+\left[\sum_{i=1}^{d}\left(M-\alpha_{i}\right)^{2}\right]^{1 / 2}$.

Therefore,

$\sqrt{M}\left\|\Theta_{1} W^{\star}-\Theta_{2} O_{0}\right\|_{F} \leq M\left\|\Omega_{1}\left[u_{1} \cdots u_{d}\right]-\Omega_{2}\left[s_{1} \cdots s_{d}\right]\right\|_{F}+2\left[\sum_{i=1}^{d}\left(M-\alpha_{i}\right)^{2}\right]^{1 / 2}$.

Now, using 60, and the principal angle property 3 , we get

$$
\left\|\Omega_{1}\left[u_{1} \cdots u_{d}\right]-\Omega_{2}\left[s_{1} \cdots s_{d}\right]\right\|_{F} \leq\left\|\sum_{i=1}^{d} \Omega_{1} u_{i}\left(\Omega_{1} u_{i}\right)^{T}-\sum_{i=1}^{d} \Omega_{2} s_{i}\left(\Omega_{2} s_{i}\right)^{T}\right\|_{F} .
$$

Moreover, using triangle inequality and properties 1 and 2, we have

$M\left\|\sum_{i=1}^{d} \Omega_{1} u_{i}\left(\Omega_{1} u_{i}\right)^{T}-\sum_{i=1}^{d} \Omega_{2} s_{i}\left(\Omega_{2} s_{i}\right)^{T}\right\|_{F} \leq\left\|W^{\star T} W^{\star}-G_{0}\right\|_{F}+\left[\sum_{i=1}^{d}\left(M-\alpha_{i}\right)^{2}\right]^{1 / 2}$.

Finally, note that by Lemma 17 .

$$
\left[\sum_{i=1}^{d}\left(M-\alpha_{i}\right)^{2}\right]^{1 / 2} \leq\left\|W^{\star T} W^{\star}-G_{0}\right\|_{F}
$$

Combining the above relations, and setting $\Theta=\Theta_{1}^{T} \Theta_{2}$, we arrive at Lemma 15 .

8.4. Proof of Lemma 16. This is done by adapting the following result by Li [42]: If $A, B$ are square and non-singular, and if $\mathcal{R}(A)$ and $\mathcal{R}(B)$ are their orthogonal rounding (obtained from their polar decompositions [32]), then

$$
\|\mathcal{R}(A)-\mathcal{R}(B)\|_{F} \leq \frac{2}{\sigma_{\min }(A)+\sigma_{\min }(B)}\|A-B\|_{F} .
$$

We recall that if $A=U \Sigma V^{T}$ is the SVD of $A$, then $\mathcal{R}(A)=U V^{T}$.

Note that it is possible that some of the blocks of $W^{\star}$ are singular, for which the above result does not hold. However, the number of such blocks can be controlled by the global error. More precisely, let $\mathcal{B} \subset\{1,2, \ldots, M\}$ be the index set such that, for $i \in \mathcal{B},\left\|W_{i}^{\star}-\Theta\right\|_{F} \geq \beta$. Then

$$
\left\|W^{\star}-\Theta O_{0}\right\|_{F}^{2} \geq \sum_{i \in \mathcal{B}}\left\|W_{i}^{\star}-\Theta\right\|_{F}^{2}=|\mathcal{B}| \beta^{2} .
$$

This gives a bound on the size of $\mathcal{B}$. In particular, the rounding error for this set can trivially be bounded as

$$
\sum_{i \in \mathcal{B}}\left\|O_{i}^{\star}-\Theta\right\|_{F}^{2} \leq \sum_{i \in \mathcal{B}} 2 d=\frac{2 d}{\beta^{2}}\left\|W^{\star}-\Theta O_{0}\right\|_{F}^{2} .
$$

On the other hand, we known that, for $i \in \mathcal{B}^{c},\left\|W_{i}^{\star}-\Theta\right\|_{F}<\beta$. From Lemma 17, it follows that

$$
\left|1-\sigma_{\min }\left(W_{i}^{\star}\right)\right| \leq\left\|W_{i}^{\star}-\Theta\right\|_{\mathrm{sp}}<\beta .
$$

Fix $\beta \leq 1$. Then $\sigma_{\min }\left(W_{i}^{\star}\right)>1-\beta$, and we have from (62),

$$
\left\|O_{i}^{\star}-\Theta\right\|_{F} \leq \frac{2}{2-\beta}\left\|W_{i}^{\star}-\Theta\right\|_{F} \quad\left(i \in \mathcal{B}^{c}\right)
$$


Fixing $\beta=1 / \sqrt{2}$ and combining (63) and (64), we get the desired bound.

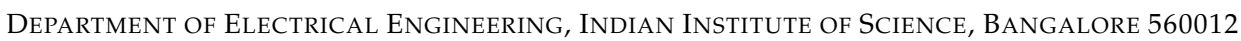

E-mail address: kunal@ee.iisc.ernet. in

Department of Physics, Princeton University, Princeton, NJ 08540

E-mail address: ykhoo@princeton.edu

Department of Mathematics And PACM, Princeton University, Princeton, NJ 08540

E-mail address: amits@math.princeton.edu 